\title{
CHAPTER NN \\ Busy period, congestion analysis and loss probability in fluid queues
}

\author{
Fabrice Guillemin ${ }^{1} \&$ Marie-Ange Remiche ${ }^{2} \&$ Bruno Sericola $^{3} *$
}

December 11, 2018

1 Orange Labs, Lannion, France

${ }^{2}$ University of Namur, Belgium

3* Inria, Rennes, France

Corresponding author, bruno.sericola@inria.fr

\begin{abstract}
Stochastic fluid flow models and in particular those driven by Markov chains have been intensively studied in the last two decades. Not only they have been proven to be efficient tools to mimic Internet traffic flow at a macroscopic level but they are useful tools in many areas of applications such as manufacturing systems or in actuarial sciences to cite but a few. This chapter proposes to focus on such a model in the context of performance analysis of a potentially congested system. The latter is modeled by means of a finite-capacity system whose content is described by a Markov driven stable fluid flow. We step-by-step describe a methodology to compute exactly the loss probability of the system. Our approach is based on the computation of hitting probabilities jointly with the peak level reached during a busy period, both in the infinite and finite buffer case. Accordingly we end up with differential Riccati equations that can be solved numerically. Moreover we are able to characterize the complete distribution of both the duration of congestion and of the total information lost during such a busy period.
\end{abstract}

Keywords: fluid queues; Markov chains; busy period; congestion; volume and duration of losses; loss probability; matrix differential Riccati equations

\section{Introduction}

In this chapter, we consider advanced queuing models arising in the performance evaluation of telecommunication networks, notably the Internet. The basic assumption underlying these models is that information flows through the 
network as a fluid. The packet nature of traffic is thus ignored: A packet stream actually appears as a fluid flow. While this assumption may be too rough at the micro-flow level (for instance a single TCP connection), it becomes very relevant when considering the superposition of a large number of micro-flows. From a mathematical point of view, this amounts to replacing discrete time arrival processes (arrivals of packets) with piecewise continuous arrival processes. Fluid flow models are very useful to study the performance of networks at a macroscopic level when aggregating a large number of flows. This is all the more relevant in current backbone networks with very high speed transmission links (above $100 \mathrm{Gbit} / \mathrm{s}$ ) capable of aggregating millions of flows. This is why fluid flow models have gained ever growing attention in the past two decades in the study of telecommunication networks, see for instance [21], [1], [2], [9], [10], [11], [15], [16] and [17].

The benefit of fluid flow models is in that it becomes easier to estimate key performance indicators characterizing the network. In particular, modern telecommunication networks heavily rely on statistical multiplexing, which allows a better usage of network capacities by exploiting the fluctuating nature of traffic flows. Typically, TCP or more recently QUIC transport protocols are based on end-to-end congestion protocols and generate sporadic flows (bursts of IP packets). Moreover, the large number of applications used today in networks (notably, video streaming) give rise to variable bit rate traffic. A direct consequence of statistical multiplexing is that a transmission link may be temporally congested. A key performance metric for characterizing statistical multiplexing in packet networks is then the volume of information lost during congestion periods of the network.

The fraction of lost information is a global performance measure reflecting congestion phenomenons occurring on each transmission link of the network when performing statistical multiplexing. In practice, several packet flows are statistically multiplexed on a same transmission link, which is in general equipped with a buffer to store packets when the arrival rate is momentarily greater than the link transmission capacity. This buffer aims at preventing as much as possible from the loss of information. However, such a buffer is necessarily with finite capacity and the arrival rate can exceed the transmission capacity for a sufficiently long time period, causing transient overflow of the buffer. Such phenomenons become more and more frequent in the current Internet since new versions of TCP or QUIC are ever more aggressive for the network; the slow start mechanism is sometimes disabled, leading to the transmission of bulks of data at very high rate.

In the following, we consider a single transmission link equipped with a buffer of finite or infinite capacity. We model the arrival of packets as a continuous process $\chi(t)$ denoting the input rate at time $t$ and we denote by $r(t)$ the net input rate at time $t$ (i.e., the difference between the instantaneous arrival and service rates). The link is congested at time $t$ when $r(t)>0$ and the buffer capacity (denoted by $x \leq \infty$ ) is reached. Denoting by $X(t)$ the volume of information in the buffer at time $t$, the fraction of lost information when $x<\infty$ 
is the long run ratio

$$
\pi_{\operatorname{loss}}=\lim _{t \rightarrow \infty} \frac{\int_{0}^{t} r(s) 1_{\{X(s)=x\}} d s}{\int_{0}^{t} \chi(s) d s}
$$

where $1_{A}$ denotes the characteristic function of the set $A$. (Note that we can have $X(t)=x$ only if $r(t) \geq 0$.) When the process $(r(t))$ is stationary and ergodic, the above equation can be rewritten as

$$
\pi_{\text {loss }}=\frac{\mathbb{E}\left(r 1_{\{X=x\}}\right)}{\mathbb{E}(\chi)},
$$

where $\chi, r$ and $X$ denote the input rate, the net input rate and the buffer occupancy in the stationary regime, respectively.

The major difficulty for estimating the quantity $\pi_{\text {loss }}$ is the computation of the joint probability distribution of the couple $(r, X)$. One classical approach consists of approximating the quantity $\pi_{\text {loss }}$ by the buffer overshoot probability $\mathbb{P}\{\bar{X}>x\}$, where $\bar{X}$ is the buffer occupancy when the buffer capacity is infinite and the system is stable (i.e., $\mathbb{E}(r)<0$ ). For large $x$, large deviations techniques can be used to approximate this latter probability.

To study the quantity $\pi_{\text {loss }}$ defined by Equation (1.1) we develop a method of computing this quantity when the input process is modulated by a Markov process. More specifically, we assume that the input rate is of the form $\chi_{\varphi(t)}$, where the phase process $(\varphi(t))$ is a Markov process with a finite state space $S$ and $\chi$ is some real valued function from $S \rightarrow \mathbb{R}$. We specifically establish the law of the volume of fluid lost during an overflow period (i.e., a time period during which the occupancy of the buffer is $x$ ) as well as the duration of such a period.

The model considered is a stochastic fluid model and in particular a Markov modulated fluid queue. These models have widely been used in several domains of system performance evaluation such as manufacturing systems, communication networks, risk processes in insurance and environmental problems, see for instance [8] and the references therein. The transient and stationary behaviors of these models have been extensively studied and numerous performance metrics with their corresponding numerical algorithms have been proposed and derived in the literature, see [12] for a long list of references on the subject.

Hitting probabilities for stochastic fluid models have been analyzed in [4, $7,8]$; the relevant algorithms have been developed in $[5,6]$. It is worth noting that the authors of [14] consider similar performance metrics via the use of Laplace transforms. The key difference with that paper is that we compute here the distribution of the random variables under consideration instead of their Laplace transforms. In particular, we compute the joint distributions of the congestion durations and the lost volumes in successive congestion periods in a same busy period. We finally prove that the total congestion duration and lost volume in a typical stationary busy period have phase type distributions. 
This chapter is divided into two main parts : In Section 2 we analyze the infinite buffer case while the finite case is treated in Section 3. Techniques developed in the infinite case will be of crucial help in the finite buffer case. Both cases are studied with the help of the hitting probabilities together with the maximum peak observed within a busy period. This permits us to obtain differential Riccati equations that can be solved numerically.

In Section 2, we first introduce the model and define the quantities of interest, such as, among others, the maximum peak or the number of such peaks observed within a busy period. These probabilities are obtained step by step and allow us to finally establish the matrix differential Riccati equations for the probability that starting at any level $u$ with an increasing buffer content, we observe at return to level $u$ a given phase and a maximum peak level less than or equal to $x+u$. We next extend our approach to the analysis of time periods when the buffer occupancy is below a given threshold.

These latter results are first extended in Section 3 to the finite buffer case. These permit us to obtain congestion metrics in terms of the complete distribution of the total lost information as well as the duration of congestion within a busy period. These finally allow us to characterize the $\pi_{\text {loss }}$ probability.

\section{Modeling a link under congestion and buffer fluctuations}

We consider in this section a classical fluid queue with infinite buffering capacity. This allows us to describe the buffer fluctuations and introduce the notation and the variables necessary to study the fluid queue when the buffer is finite.

\subsection{Model description}

The input and service rates are controlled by a homogeneous Markov chain $\varphi=(\varphi(t), t \geq 0)$ taking values in the finite state space $S$, with infinitesimal generator $T$ and initial probability distribution $\alpha$. The process $\varphi$ is also called the phase process and we denote by $X(t)$ the amount of fluid in the buffer at time $t$. It is well-known that the process $((X(t), \varphi(t)), t \geq 0)$ forms a Markov process having a pair of continuous and discrete states.

Let $\chi_{i}$ be the input rate and $\eta_{i}$ be the service rate of the queue when the Markov chain $\varphi$ is in state $i$. We denote by $r_{i}$ the net (or effective) input rate of state $i$, that is $r_{i}=\chi_{i}-\eta_{i}$ and we define the diagonal matrix of net input rates $R=\operatorname{diag}\left(r_{i}, i \in S\right)$. We denote by $S^{0}, S^{-}$and $S^{+}$the subsets of states $i \in S$ such that $r_{i}=0, r_{i}<0$ and $r_{i}>0$, respectively. In the same way, we denote by $R_{0}, R_{-}$and $R_{+}$the diagonal matrices $R_{0}=\operatorname{diag}\left(r_{i}, i \in S^{0}\right)$, $R_{-}=\operatorname{diag}\left(-r_{i}, i \in S^{-}\right)$and $R_{+}=\operatorname{diag}\left(r_{i}, i \in S^{+}\right)$. We clearly have $R_{0}=0$, the null matrix.

The numbers of states in $S^{0}, S^{+}$and $S^{-}$are denoted by $n_{0}, n_{+}$and $n_{-}$, respectively. By using this partition of states, we decompose the transition 
matrix $T$ as

$$
T=\left(\begin{array}{ccc}
T_{--} & T_{-0} & T_{-+} \\
T_{0-} & T_{00} & T_{0+} \\
T_{+-} & T_{+0} & T_{++}
\end{array}\right) .
$$

The Markov chain $\varphi$ is supposed to be irreducible and we denote by $p=$ $\left(p_{i}, i \in S\right)$ its stationary distribution. We thus have $p T=0$ and $p \mathbb{1}=1$, where $\mathbb{1}$ is the column vector with all entries equal to 1 ; its dimension is specified by the context.

We suppose that the stability condition for the fluid queue is satisfied, which means that

$$
\lim _{t \rightarrow \infty} \mathbb{E}\left(r_{\varphi(t)}\right)=\sum_{i \in S} r_{i} p_{i}<0 .
$$

This condition ensures that all the busy periods are of finite length a.s. and that the maximum level of the queue during every busy period is finite a.s., in the infinite buffer case.

Let us introduce the $\left(n_{-}+n_{+}\right) \times\left(n_{-}+n_{+}\right)$matrix

$$
Q=\left(\begin{array}{ll}
Q_{--} & Q_{-+} \\
Q_{+-} & Q_{++}
\end{array}\right)
$$

where $Q_{--}=R_{-}^{-1}\left(T_{--}-T_{-0} T_{00}^{-1} T_{0-}\right), Q_{-+}=R_{-}^{-1}\left(T_{-+}-T_{-0} T_{00}^{-1} T_{0+}\right)$, $Q_{+-}=R_{+}^{-1}\left(T_{+-}-T_{+0} T_{00}^{-1} T_{0-}\right)$ and $Q_{++}=R_{+}^{-1}\left(T_{++}-T_{+0} T_{00}^{-1} T_{0+}\right)$.

These matrices allow us to restrict our problem to a Markov chain with state space $S^{-} \cup S^{+}$with infinitesimal generator $Q$ and effective input rates equal to -1 or 1 . For instance the matrix $Q_{--}$governs the transitions from $i \in S^{-}$to $j \in S^{-}$without any visit to $S^{+}$. More formally, as shown in [19] and [4], $e^{Q_{--} x}(i, j)$ is the probability, starting from state $i$, to reach state $j$ with an accumulated reward equal to $x$ and without leaving the set $S^{0} \cup S^{-}$. Here the accumulated reward corresponds to the amount of fluid generated from the effective input rates of the matrix $R_{-}$. A symmetric interpretation holds for matrix $Q_{++}$. Concerning the matrix $Q_{-+}$, the entry $Q_{-+}(i, j)$ is the rate, rescaled according to matrix $R_{-}$, at which state $j$ is reached from state $i$ either directly from state $i$ or after some time spent in subset $S^{0}$. A symmetric interpretation holds for matrix $Q_{+-}$. Such a transformation consists in considering that the time spent in the zero rate states is immaterial.

Since we are concerned by quantities such as the minimum valley, the maximum level and hitting probabilities, the fluid queue with parameters $(T, R)$ is equivalent to the fluid queue with parameters $(Q, C)$ where $C$ is the $\left(n_{-}+n_{+}\right) \times$ $\left(n_{-}+n_{+}\right)$matrix

$$
C=\left(\begin{array}{cc}
-I & 0 \\
0 & I
\end{array}\right)
$$

where $I$ is the identity matrix whose dimension is specified by the context of its use. This transformation has been also suggested in [3] and [18].

In view of the above observations, we will consider in the following a fluid queue driven by a Markov chain $\varphi=(\varphi(t), t \geq 0)$ with state space $S=S^{-} \cup S^{+}$, 
infinitesimal generator $Q$ and effective input rates given by matrix $C$, i.e. equal to -1 or 1 .

\subsection{Peaks and valleys}

Let us consider a busy period of a fluid queue. The buffer occupancy $X(t)$ is piecewise linear as illustrated in Figure 1a with peaks followed by valleys. Let $M$ be the maximum peak level in the queue during a busy period. We moreover denote by $H$ the fluid level at the minimum valley and by $L$ the integer-valued random variable representing the index of the smallest valley, when valleys are numbered in the order of their occurrence, if they exist. Note that a busy period can have a single peak as illustrated in Figure 1b.

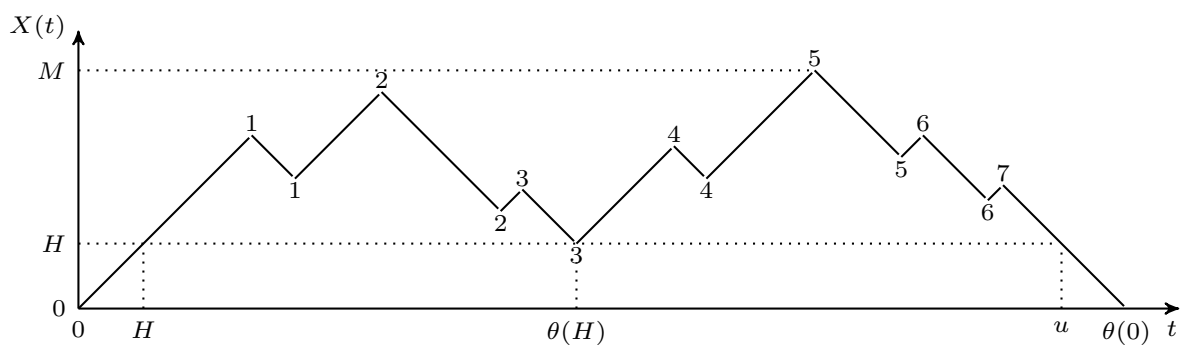

a) A busy period with valleys $(u=\theta(0)-H)$

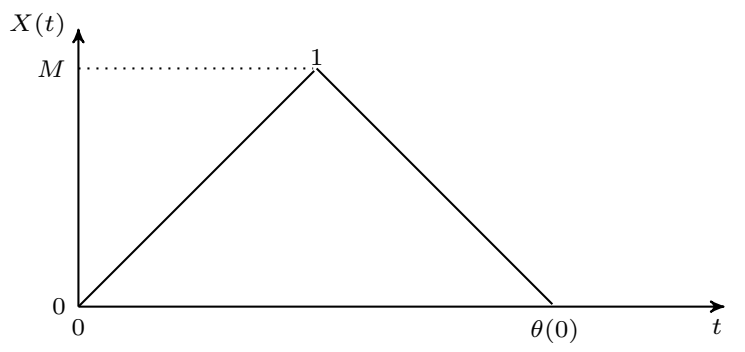

b) A busy period without valley

Figure 1: A classical busy period.

For any $x \geq 0$, let $\theta(x)$ denote the first instant greater than $x$ at which the fluid level is equal to $x$, that is,

$$
\theta(x)=\inf \{t>x \mid X(t)=x\} .
$$

When $X(0)=0$ and $\varphi(0) \in S^{-}$then, by definition, we have $\theta(0)=0$.

When $X(0)=0$ and $\varphi(0) \in S^{+}$, the instants 0 and $\theta(0)$ are respectively the initial and the final instants of the busy period. These variables are shown in Figure 1 in the case where the effective input rates are either equal to -1 
or 1 . We have numbered the successive peaks and valleys arising during a busy period.

For every $n \geq 2, \ell=1, \ldots, n-1$ and $x \geq 0$, we consider the $n_{+} \times n_{-}$matrix $F(n, \ell, y)$ whose entries are defined, for $i \in S^{+}$and $j \in S^{-}$, by

$$
F_{i, j}(n, \ell, y)=\mathbb{P}\{\varphi(\theta(0))=j, N=n, L=\ell, H \leq y \mid \varphi(0)=i, X(0)=0\},
$$

where $N$ is the number of peaks arising during a busy period. In the case $N=1$, as shown in Figure 1b, there is only one peak during the busy period and so, since there is no valley, we do not define the matrix for $n=1$. In the path described in Figure 1a, we have $N=7$ and $L=3 . F_{i, j}(\ell, n, y)$ is the probability that a busy period, starting in phase $i$, contains $n$ peaks, has its minimum valley index equal to $\ell$, its minimum valley less than or equal to $x$ and ends in phase $j$.

We also consider the $n_{+} \times n_{-}$matrix $\Psi(x, y)$ whose entries are defined, for $i \in S^{+}, j \in S^{-}, x \geq 0$ and $0 \leq y \leq x$, by

$$
\Psi_{i, j}(x, y)=\mathbb{P}\{\varphi(\theta(0))=j, M \leq x, H \leq y \mid \varphi(0)=i, X(0)=0\},
$$

and the $n_{-} \times n_{+}$matrix $\Theta(x)$ whose entries are defined, for $i \in S^{-}, j \in S^{+}$and $x>0$, by

$$
\Theta_{i, j}(x)=\mathbb{P}\{\varphi(\gamma(x))=j \mid \varphi(0)=i, X(0)=x\},
$$

where, for any $x \geq 0, \gamma(x)$ denotes the first positive instant at which the fluid level is equal to $x$, i.e.

$$
\gamma(x)=\inf \{t>0 \mid X(t)=x\} .
$$

The quantity $\Psi_{i, j}(x, y)$ is the probability that a busy period, starting in phase $i$, has its maximum peak less than or equal to $x$, its minimum valley less than or equal to $y$ and ends in phase $j . \Theta_{i, j}(x)$ is the probability that a busy period, starting in phase $i$, ends in phase $j$.

Note that $\gamma(x)$ is the first time at which the fluid level is equal to $x$. The function $\theta(x)$ is used to detect the time at which the minimum valley $H$ when $X_{0}=0$ (see Figure 1a) or the maximum peak $M$ when $X_{0}>0$ (see Figure 2a) occur.

By definition of $H$ and $M$, when $X_{0}=0, \theta(H)$ is the second instant at which the fluid level is equal to $H$ (see Figure 1a) and, when $X_{0}>0, \theta(M)$ is the second instant at which the fluid level is equal to $M$ (see Figure 2a).

\subsection{Minimum valley height in a busy period}

For $i \in S^{+}, j \in S^{-}, n \geq 2, \ell=1, \ldots, n-1$ and $y \geq 0$, we denote by $f_{i, j}(n, \ell, y)$ the joint density associated with the distribution $F_{i, j}(n, \ell, y)$ given by Equation $(2.2)$ and defined by $f_{i, j}(n, \ell, y)=d F_{i, j}(n, \ell, y) / d y$. We also introduce, for $n \geq 1$, the $n_{+} \times n_{-}$matrix $W(n)$ whose entries are defined, for $i \in S^{+}$and $j \in S^{-}$, by

$$
W_{i, j}(n)=\mathbb{P}\{\varphi(\theta(0))=j, N=n \mid \varphi(0)=i, X(0)=0\} .
$$




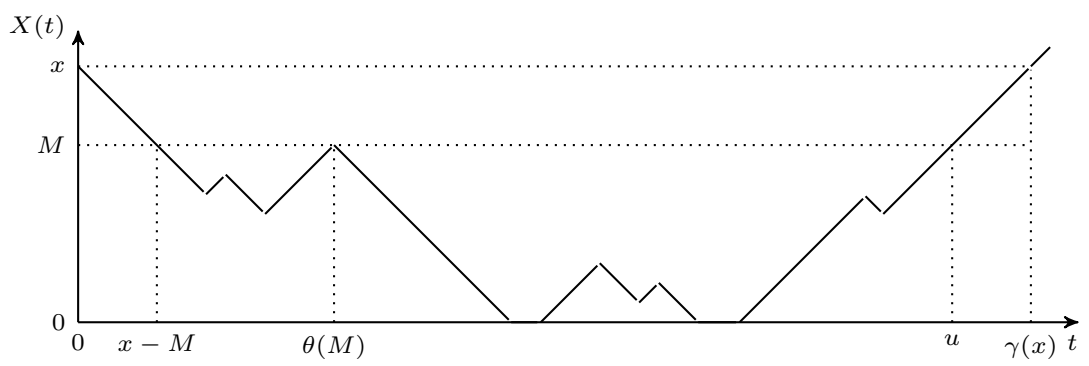

a) with at least 2 valleys $(u=\gamma(x)-x+M)$

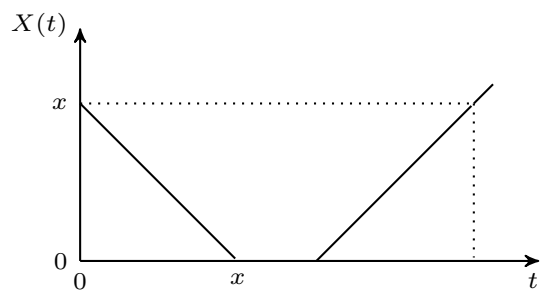

b) without valley

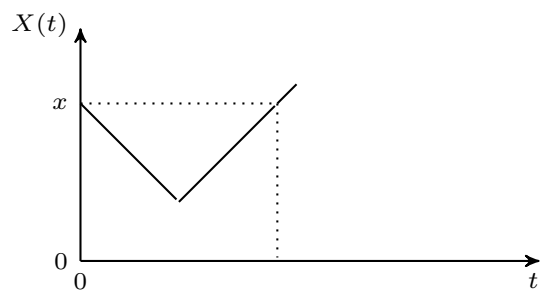

c) with only one valley

Figure 2: A period under level $x$.

The quantity $W_{i, j}(n)$ is the probability that a busy period, starting in phase $i$, contains $n$ peaks and ends in phase $j$. Summing and integrating the density $f_{i, j}(\ell, n, y)$ over the location and the value of the minimum valley, we obtain, for $n \geq 2$,

$$
W_{i, j}(n)=\int_{0}^{\infty} \sum_{\ell=1}^{n-1} f_{i, j}(n, \ell, y) d y .
$$

The $n_{+} \times n_{-}$matrix containing the terms $f_{i, j}(n, \ell, y)$ is denoted by $f(n, \ell, y)$ and is given together with matrix $W(1)$ by the following theorem.

Theorem 1. We have

$$
W(1)=\int_{0}^{\infty} e^{Q_{++} y} Q_{+-} e^{Q_{--} y} d y
$$

and for $n \geq 2$

$$
W(n)=\int_{0}^{\infty} e^{Q_{++} y} \sum_{\ell=1}^{n-1} W(\ell) Q_{-+} W(n-\ell) e^{Q_{--} y} d y
$$

Proof. The proof follows the same arguments as those developed in [4]. To obtain the expression for $W(1)$ we consider a sample path starting from fluid level 0 in phase $i \in S^{+}$and returning to level 0 in phase $j \in S^{-}$with only one 
peak of height $y$. Such a sample path (see Figure 1b) can be decomposed into three stages as follows :

1. The phase process $\varphi$ starts in phase $i \in S^{+}$and reaches some state $k \in S^{+}$ at time $y$ without leaving subset $S^{+}$. This means that the fluid level increases from level 0 to level $y$. As seen in the previous section, the corresponding probability is equal to $e^{Q_{++} y}(i, k)$.

2. Since a peak (of height equal to $y$ ) occurs, this means that a transition of the phase process $\varphi$ occurs from state $k$ to some state $h \in S^{-}$. The corresponding transition rate is equal to $Q_{+-}(k, h)$.

3. Starting from state $h \in S^{-}$, the process $\varphi$ reaches state $j \in S^{-}$at time $y$ without leaving subset $S^{-}$. This means that the fluid level decreases from level $y$ to level 0 . As seen in the previous section, the corresponding probability is equal to $e^{Q--y}(h, j)$.

We thus obtain

$$
W_{i, j}(1)=\int_{0}^{\infty} \sum_{k \in S^{+}} \sum_{h \in S^{-}} e^{Q_{++} y}(i, k) Q_{+-}(k, h) e^{Q_{--}}(h, j) d y,
$$

that is

$$
W(1)=\int_{0}^{\infty} e^{Q_{++} y} Q_{+-} e^{Q_{--}} d y .
$$

Let now $n \geq 2$ and $1 \leq \ell \leq n-1$. A typical example of such a sample path is shown in Figure 1a. It is a path starting from fluid level 0 in phase $i \in S^{+}$ and returning to level 0 in phase $j \in S^{-}$with $N=n$ peaks, with the minimum valley of height $H=y$ at position $L=\ell$. Such a sample path can be broken up into five stages as follows :

1. As we can observe, $H$ denotes the minimum level as well as the amount of time needed to reach it. The phase process $\varphi$ starts in phase $i \in S^{+}$ and reaches some state $k \in S^{+}$at time $H=y$ without leaving subset $S^{+}$. This means that the fluid level increases from level 0 to level $y$. As seen in the previous section, the corresponding probability is equal to $e^{Q_{++} y}(i, k)$.

2. Starting from phase $k \in S^{+}$and level $y$, the fluid process returns for the first time to level $y$ in some phase $h \in S^{-}$. By definition of $H$ and $L$, the number of peaks between instants $H$ and $\theta(H)$ is equal to $\ell$. By the spatial homogeneity of the process, the corresponding probability is equal to $W_{k, h}(\ell)$.

3. Since a valley (of height equal to $y$ at time $\theta(H)$ ) occurs, this means that a transition of the phase process $\varphi$ occurs from state $h \in S^{-}$to some state $m \in S^{+}$. The corresponding transition rate is equal to $Q_{-+}(h, m)$. 
4. Once again, starting from phase $m \in S^{+}$and level $y$, the fluid process returns for the first time to level $y$ in some phase $v \in S^{-}$. By definition of $H$ and $L$, the number of peaks between instants $\theta(H)$ and $\theta(0)-H$ is equal to $n-\ell$. By the spatial homogeneity of the process, the corresponding probability is equal to $W_{m, v}(n-\ell)$.

5. The phase process $\varphi$ starts in phase $v \in S^{-}$and level $y$. It reaches level 0 in state $j$ without leaving subset $S^{-}$, at time $\varphi(\theta(0))$. This means that the fluid level decreases from level $y$ to level 0 . As seen in the previous section, the corresponding probability is equal to $e^{Q_{--} y}(v, j)$.

We thus obtain

$$
\sum_{k \in S^{+}} \sum_{h \in S^{-}} \sum_{m \in S^{+}} \sum_{v \in S^{-}} e^{Q_{++} y}(i, k) W_{k, h}(\ell) Q_{-+}(h, m) W_{m, v}(n-\ell) e^{Q_{--}}(v, j),
$$

that is $f(n, \ell, y)=e^{Q_{++} y} W(\ell) Q_{-+} W(n-\ell) e^{Q_{--} y}$. Equation (2.6) follows by using Equation (2.4).

Let $\Psi$ denote $n_{+} \times n_{-}$the stochastic matrix whose entries are defined, for $i \in S^{+}$and $j \in S^{-}$, by $\Psi_{i, j}=\mathbb{P}\{\varphi(\theta(0))=j \mid \varphi(0)=i, X(0)=0\} . \Psi_{i, j}$ is the probability that the phase at the instant of the first return to the initial level is equal to $j$. By spatial homogeneity, this probability is independent of the value of the initial level. We have

$$
\Psi=\sum_{n=1}^{\infty} W(n)
$$

In the next theorem, we give a relation satisfied by matrix $\Psi$.

\section{Theorem 2.}

$$
\Psi=\int_{0}^{\infty} e^{Q_{++} y} Q_{+-} e^{Q_{--} y} d y+\int_{0}^{\infty} e^{Q_{++} y} \Psi Q_{-+} \Psi e^{Q_{--} y} d y .
$$

Proof. From Equation (2.6), we have for $n \geq 2$

$$
W(n)=\int_{0}^{\infty} e^{Q_{++} y} \sum_{\ell=1}^{n-1} W(\ell) Q_{-+} W(n-\ell) e^{Q_{--} y} d y .
$$


By summation over $n$, we get, using the monotone convergence theorem,

$$
\begin{aligned}
\Psi & =W(1)+\sum_{n=2}^{\infty} \int_{0}^{\infty} e^{Q_{++} y} \sum_{\ell=1}^{n-1} W(\ell) Q_{-+} W(n-\ell) e^{Q_{--y}} d y \\
& =W(1)+\int_{0}^{\infty} e^{Q_{++} y} \sum_{n=2}^{\infty} \sum_{\ell=1}^{n-1} W(\ell) Q_{-+} W(n-\ell) e^{Q_{--} y} d y \\
& =W(1)+\int_{0}^{\infty} e^{Q_{++} y} \sum_{\ell=1}^{\infty} \sum_{n=\ell+1}^{\infty} W(\ell) Q_{-+} W(n-\ell) e^{Q_{--} y} d y \\
& =W(1)+\int_{0}^{\infty} e^{Q_{++} y} \sum_{\ell=1}^{\infty} W(\ell) Q_{-+} \sum_{n=\ell+1}^{\infty} W(n-\ell) e^{Q_{--} y} d y \\
& =W(1)+\int_{0}^{\infty} e^{Q_{++} y} \Psi Q_{-+} \Psi e^{Q_{--} y} d y,
\end{aligned}
$$

which is the desired result.

It is easily checked that matrix $\Psi$ is solution to the following matrix algebraic Riccati equation $Q_{++} \Psi+\Psi Q_{--}+\Psi Q_{-+} \Psi+Q_{+-}=0$. This equation has been considered in several papers and several algorithms have been developed to compute $\Psi$ which is the minimal solution of this equation, see for instance [4], $[5]$ and the references therein. An explicit expression for $\Psi$ has been obtained in [20] by means of an auxiliary matrix.

Let $F(x)$ denote the $n_{+} \times n_{-}$matrix whose entries are defined, for $i \in S^{+}$ and $j \in S^{-}$, by $F_{i, j}(x)=\mathbb{P}\{\varphi(\theta(0))=j, H \leq x \mid \varphi(0)=i\} . F_{i, j}(x)$ is the probability that the phase at the instant of the first return to the initial level is equal to $j$ with a minimum valley of height less than or equal to $x$. This probability is defined only when the minimum valley exists. The minimum valley exists if and only if the number of peaks $N$ is greater than or equal to 2 . Moreover, when it exists, we have $H>0$. Thus we define the event $H=0$ to represent the non-existence of the minimum valley, i.e. the case where the number of peaks is equal to 1 (see Figure 1b). We then have the following result.

Corollary 3. For every $x \geq 0$,

$$
F(x)=\int_{0}^{\infty} e^{Q_{++} y} Q_{+-} e^{Q_{--} y} d y+\int_{0}^{x} e^{Q_{++} y} \Psi Q_{-+} \Psi e^{Q_{--} y} d y .
$$

Proof. By definition, we have, for $i \in S^{+}$and $j \in S^{-}$,

$$
\begin{aligned}
& F_{i, j}(x) \\
& =\mathbb{P}\{\varphi(\theta(0))=j, H=0 \mid \varphi(0)=i\}+\mathbb{P}\{\varphi(\theta(0))=j, 0<H \leq x \mid \varphi(0)=i\} \\
& =\mathbb{P}\{\varphi(\theta(0))=j, N=1 \mid \varphi(0)=i\}+\mathbb{P}\{\varphi(\theta(0))=j, N \geq 2, H \leq x \mid \varphi(0)=i\} \\
& =W_{i, j}(1)+\int_{0}^{x} \sum_{n=2}^{\infty} \sum_{\ell=1}^{n-1} f_{i, j}(n, \ell, y) d y .
\end{aligned}
$$

Following the same lines used in the proof of Theorem 2, we get the result. 


\subsection{Maximum peak level in a busy period}

We consider in this section the maximum fluid level $M$ reached during a busy period. A typical path is shown in Figure 1. For $i \in S^{+}, j \in S^{-}, x \geq 0$ and $0 \leq y \leq x$, we denote by $\psi_{i, j}(x, y)$ the marginal density associated with the distribution

$$
\Psi_{i, j}(x, y)=\mathbb{P}\{\varphi(\theta(0))=j, M<x, H \leq y \mid \varphi(0)=i, X(0)=0\},
$$

and defined by $\psi_{i, j}(x, y)=\partial \Psi_{i, j}(x, y) / \partial y$. For $x \geq 0$, we denote by $\Psi(x)$ the $n_{+} \times n_{-}$matrix containing the $\Psi_{i, j}(x)$ defined, for $i \in S^{+}$and $j \in S^{-}$, by

$$
\Psi_{i, j}(x)=\mathbb{P}\{\varphi(\theta(0))=j, M \leq x \mid \varphi(0)=i, X(0)=0\} .
$$

$\Psi_{i, j}(x)$ is the probability that, starting from phase $i \in S^{+}$and any level $u \geq 0$, the phase at the instant of the first return to the initial level $u$ is equal to $j$ and the maximum level is less than or equal to $x+u$. By the spatial homogeneity, this probability is independent of $u$, that is why we define $\Psi(x)$ only for $u=0$. The $n_{+} \times n_{-}$matrix containing the terms $\psi_{i, j}(x, y)$ is denoted by $\psi(x, y)$ and is given by the following theorem.

Theorem 4. For $x \geq 0$ and $0 \leq z \leq x$, we have

$$
\begin{aligned}
& \Psi(x, z)=\int_{0}^{x} e^{Q_{++} y} Q_{+-} e^{Q_{--} y} d y+\int_{0}^{z} e^{Q_{++} y} \Psi(x-y) Q_{-+} \Psi(x-y) e^{Q_{--} y} d y . \\
& \Psi(x)=\int_{0}^{x} e^{Q_{++} y} Q_{+-} e^{Q_{--} y} d y+\int_{0}^{x} e^{Q_{++} y} \Psi(x-y) Q_{-+} \Psi(x-y) e^{Q_{--} y} d y .
\end{aligned}
$$

Proof. We proceed as for the proof of Theorem 1 . The term $\Psi(x, 0)$ corresponds to the case where there is only one peak (see Figure $1 \mathrm{~b}$ ), and thus no valley, which means, as defined in the previous section, that $H=0$. To obtain the expression for $\Psi(x, 0)$ we consider a sample path starting from fluid level 0 in phase $i \in S^{+}$and returning to level 0 in phase $j \in S^{-}$with only one peak of height $y$ (with $y \leq x$ ). Such a sample path can be broken up into three stages as follows :

1. The phase process $\varphi$ starts in phase $i \in S^{+}$and reaches some state $k \in S^{+}$ at time $y$ without leaving subset $S^{+}$. This means that the fluid level increases from level 0 to level $y$. As seen in the previous section, the corresponding probability is equal to $e^{Q_{++} y}(i, k)$.

2. Since a peak (of height equal to $y$ ) occurs, this means that a transition of the phase process $\varphi$ occurs from state $k$ to some state $h \in S^{-}$. The corresponding transition rate is equal to $Q_{+-}(k, h)$.

3. Starting from state $h \in S^{-}$, the process $\varphi$ reaches state $j \in S^{-}$at time $y$ without leaving subset $S^{-}$. This means that the fluid level decreases from level $y$ to level 0 . As seen in the previous section, the corresponding probability is equal to $e^{Q_{--y}}(h, j)$. 
We thus obtain

$$
\Psi_{i, j}(x, 0)=\int_{0}^{x} \sum_{k \in S^{+}} \sum_{h \in S^{-}} e^{Q_{++} y}(i, k) Q_{+-}(k, h) e^{Q_{--}}(h, j) d y,
$$

that is

$$
\Psi(x, 0)=\int_{0}^{x} e^{Q_{++} y} Q_{+-} e^{Q_{--}} d y .
$$

We consider now the case where a minimum valley exists, i.e. $H>0$. A typical example of such a sample path is shown in Figure 1a. It is a path starting from fluid level 0 in phase $i \in S^{+}$and returning to level 0 in phase $j \in S^{-}$with at least two peaks (i.e. $H>0$ ), with the minimum valley and the maximum level less than or equal to $x$. Such a sample path can be broken up into five stages as follows :

1. The phase process $\varphi$ starts in phase $i \in S^{+}$and reaches some state $k \in S^{+}$ at time $H=y$ (with $y \leq x$ ) without leaving subset $S^{+}$. This means that the fluid level increases from level 0 to level $y$. As seen in the previous section, the corresponding probability is equal to $e^{Q_{++} y}(i, k)$.

2. Starting from phase $k \in S^{+}$and level $y$, the fluid process returns for the first time to level $y$ in some phase $h \in S^{-}$, without exceeding level $x$. By the spatial homogeneity of the process, the corresponding probability is equal to $\Psi(x-y)$.

3. Since a valley (of height equal to $y$ at time $\theta(H)$ ) occurs, this means that a transition of the phase process $\varphi$ occurs from state $h \in S^{-}$to some state $m \in S^{+}$. The corresponding transition rate is equal to $Q_{-+}(h, m)$.

4. Once again, starting from phase $m \in S^{+}$and level $y$, the fluid process returns for the first time to level $y$ in some phase $v \in S^{-}$without exceeding level $x$. By the spatial homogeneity of the process, the corresponding probability is equal to $\Psi(x-y)$.

5. The phase process $\varphi$ starts in phase $v \in S^{-}$and level $y$. It reaches level 0 in state $j$ without leaving subset $S^{-}$, at time $\varphi(\theta(0))$. This means that the fluid level decreases from level $y$ to level 0 . As seen in the previous section, the corresponding probability is equal to $e^{Q_{--y}}(v, j)$.

We thus obtain

$$
\begin{aligned}
& \psi_{i, j}(x, y)= \\
& \sum_{k \in S^{+}} \sum_{h \in S^{-}} \sum_{m \in S^{+}} \sum_{v \in S^{-}} e^{Q_{++} y}(i, k) \Psi_{k, h}(x-y) Q_{-+}(h, m) \Psi_{m, v}(x-y) e^{Q_{--y}}(v, j),
\end{aligned}
$$


which can be rewritten as $\psi(x, y)=e^{Q_{++} y} \Psi(x-y) Q_{-+} \Psi(x-y) e^{Q_{--} y}$. Equation (2.8) follows by writing

$$
\Psi(x, z)=\Psi(x, 0)+\int_{0}^{z} \psi(x, y) d y .
$$

Since $\Psi(x)=\Psi(x, x)$, Equation (2.9) follows.

We have $\lim _{x \longrightarrow \infty} \Psi(x, z)=F(z)$ and $\lim _{x \rightarrow \infty} \Psi(x)=\Psi$. We denote by $\Psi^{\prime}(x)$ the derivative of $\Psi(x)$ with respect to $x$. We then have the following result.

Theorem 5. The function $\Psi(x)$ satisfies the following matrix differential Riccati equation

$$
\Psi^{\prime}(x)=Q_{++} \Psi(x)+\Psi(x) Q_{--}+\Psi(x) Q_{-+} \Psi(x)+Q_{+-},
$$

with $\Psi(0)=0$ as initial condition.

Proof. By definition of function $\Psi(x)$ in Relation (2.7), the initial condition is trivially given by $\Psi(0)=0$, since the maximum level $M$ during a busy period is positive. Using a variable change, Relation (2.9) can be written as

$$
\Psi(x)=\int_{0}^{x} e^{Q_{++} y} Q_{+-} e^{Q_{--} y} d y+\int_{0}^{x} e^{Q_{++}(x-y)} \Psi(y) Q_{-+} \Psi(y) e^{Q_{--}(x-y)} d y .
$$

In order to avoid too long expressions in the derivation of $\Psi^{\prime}(x)$, we introduce the following notation

$\alpha(x)=\int_{0}^{x} e^{Q_{++} y} Q_{+-} e^{Q_{--} y} d y$ and $\beta(x)=\int_{0}^{x} e^{-Q_{++} y} \Psi(y) Q_{-+} \Psi(y) e^{-Q_{--y}} d y$.

It is easy to check that $e^{Q_{++} x} Q_{+-} e^{Q_{--} x}-Q_{++} \alpha(x)-\alpha(x) Q_{--}=Q_{+-}$. Differentiating (2.9), we get

$$
\begin{aligned}
\Psi^{\prime}(x)= & e^{Q_{++} x} Q_{+-} e^{Q_{--}}+Q_{++} e^{Q_{++} x} \beta(x) e^{Q_{--} x} \\
& +e^{Q_{++} x}\left[\beta^{\prime}(x) e^{Q_{--} x}+\beta(x) e^{Q_{--} x} Q_{--}\right] \\
= & e^{Q_{++} x} Q_{+-} e^{Q_{--} x}+Q_{++}[\Psi(x)-\alpha(x)]+e^{Q_{++} x} \beta^{\prime}(x) e^{Q_{--} x} \\
& +[\Psi(x)-\alpha(x)] Q_{--} \\
= & Q_{++} \Psi(x)+\Psi(x) Q_{--}+e^{Q_{++} x} \beta^{\prime}(x) e^{Q_{--} x}+Q_{+-} \\
= & Q_{++} \Psi(x)+\Psi(x) Q_{--}+\Psi(x) Q_{-+} \Psi(x)+Q_{+--},
\end{aligned}
$$

which is the desired result.

With the initial condition $\Psi(0)=0$, the Cauchy-Lipschitz Theorem ensures that the matrix differential Riccati equation (2.10) has a unique solution. This solution is thus given, for $x \geq 0$, by (2.7). By definition of diagonal matrix $C$, we have

$$
C Q=\left(\begin{array}{cc}
-Q_{--} & -Q_{-+} \\
Q_{+-} & Q_{++}
\end{array}\right) .
$$


According to the decomposition $S=S^{-} \cup S^{+}$, we define the four matrices $A(x)$, $B(x), C(x)$ and $D(x)$ occurring in the matrix $e^{C Q x}$ by writing

$$
e^{C Q x}=\left(\begin{array}{cc}
A(x) & B(x) \\
C(x) & D(x)
\end{array}\right)
$$

The following theorem gives an expression of the solution $\Psi(x)$ to the matrix differential Riccati equation (2.10).

Theorem 6. For every $x \geq 0$, we have $\Psi(x)=C(x) A(x)^{-1}$.

Proof. Let us consider the following linear differential equation

$$
Y^{\prime}(x)=\left(-Q_{--}-Q_{-+} \Psi(x)\right) Y(x) \text { with } Y(0)=I .
$$

The function $\Psi$ being continuous, this linear system has a unique solution which is invertible. We now define the $n_{+} \times n_{-}$matrix $Z(x)$ by $Z(x)=\Psi(x) Y(x)$. Using this definition, equation (2.12) becomes

$$
Y^{\prime}(x)=-Q_{--} Y(x)-Q_{-+} Z(x) .
$$

Differentiating $Z(x)$ with respect to $x$, we obtain from (2.10) and (2.13)

$$
\begin{aligned}
Z^{\prime}(x)= & \Psi^{\prime}(x) Y(x)+\Psi(x) Y^{\prime}(x) \\
= & \left(Q_{++} \Psi(x)+\Psi(x) Q_{--}+\Psi(x) Q_{-+} \Psi(x)+Q_{+-}\right) Y(x) \\
& +\Psi(x)\left(-Q_{--} Y(x)-Q_{-+} Z(x)\right) \\
= & Q_{++} Z(x)+\Psi(x) Q_{-+} \Psi(x) Y(x)+Q_{+-} Y(x)-\Psi(x) Q_{-+} Z(x) \\
= & Q_{++} Z(x)+Q_{+-} Y(x) .
\end{aligned}
$$

Putting together equations (2.13) and (2.14) we obtain

$$
\left(\begin{array}{c}
Y^{\prime}(x) \\
Z^{\prime}(x)
\end{array}\right)=\left(\begin{array}{cc}
-Q_{--} & -Q_{-+} \\
Q_{+-} & Q_{++}
\end{array}\right)\left(\begin{array}{c}
Y(x) \\
Z(x)
\end{array}\right)=C Q\left(\begin{array}{c}
Y(x) \\
Z(x)
\end{array}\right),
$$

with $Y(0)=I$ and $Z(0)=0$. The solution to that equation is given by

$$
\left(\begin{array}{c}
Y(x) \\
Z(x)
\end{array}\right)=e^{C Q x}\left(\begin{array}{c}
I \\
0
\end{array}\right)=\left(\begin{array}{c}
A(x) \\
C(x)
\end{array}\right),
$$

which means that $Y(x)=A(x), Z(x)=C(x)$ and thus, since $A(x)$ is invertible, we have $\Psi(x)=C(x) A(x)^{-1}$.

\subsection{Maximum peak under a fixed fluid level}

In this section, we still consider an infinite fluid queue and we are interested in time periods when the buffer occupancy is below a given threshold $x$.

We consider the first return to the initial level $x$ when $x>0$ and the initial phase is in $S^{-}$. More formally, we introduce the $n_{-} \times n_{+}$matrix $G(x, y)$ whose entries are defined, for $i \in S^{-}, j \in S^{+}, x>0$ and $0 \leq y \leq x$, by

$$
G_{i, j}(x, y)=\mathbb{P}\{\varphi(\gamma(x))=j, M \leq y \mid \varphi(0)=i, X(0)=x\},
$$


where $M$ denotes the maximum peak between instants 0 and $\gamma(x)$. When there are no peaks, we take as convention $M=0$. The $n_{-} \times n_{+}$matrix $\Theta(x)$ whose entries are defined, for $i \in S^{-}, j \in S^{+}$and $x>0$, by

$$
\Theta_{i, j}(x)=\mathbb{P}\{\varphi(\gamma(x))=j \mid \varphi(0)=i, X(0)=x\},
$$

satisfies $\Theta(x)=G(x, x)$. By spatial homogeneity, we have, for $i \in S^{+}$and $j \in S^{-}, \mathbb{P}\{\varphi(\gamma(x))=j \mid \varphi(0)=i, X(0)=x\}=\Psi_{i, j}$. For $i \in S^{-}$and $j \in S^{+}$, the problem is more complicated because of the influence of the boundary level zero which makes the hitting probability $\Theta_{i, j}(x)$ dependent of $x$. A typical example of such a sample path is shown in Figure 2.

We denote by $g_{i, j}(x, y)$ the density associated with the distribution $G_{i, j}(x, y)$, i.e. $g_{i, j}(x, y)=\partial G_{i, j}(x, y) / \partial y$.

Theorem 7. For $x>0$, we have

$$
G(x, 0)=\int_{0}^{x} e^{Q_{--} y} Q_{-+} e^{Q_{++} y} d y+\left(-Q_{--}\right)^{-1} e^{Q_{--} x} Q_{-+} e^{Q_{++} x}
$$

and, for $0 \leq y \leq x$,

$$
g(x, y)=e^{Q_{--} y} \Theta(x-y) Q_{+-} \Theta(x-y) e^{Q_{++} y} .
$$

Proof. We proceed as for the proof of Theorems 1 and 4 . The term $G(x, 0)$ corresponds to the case where there are no peaks in the interval $[0, \gamma(x)]$, i.e. where $M=0$. This situation corresponds to the paths shown in Figures $2 \mathrm{~b}$ and 2c. Figure 2b corresponds to the case where the height of the unique valley, denoted by $H$, is zero and Figure 2c corresponds to the case where $H$ is positive. We thus have

$$
\begin{aligned}
G(x, 0)= & \mathbb{P}\{\varphi(\gamma(x))=j, M=0, H>0 \mid \varphi(0)=i, X(0)=x\} \\
& +\mathbb{P}\{\varphi(\gamma(x))=j, M=0, H=0 \mid \varphi(0)=i, X(0)=x\} .
\end{aligned}
$$

The first term is the symmetric term of $\Psi(x, 0)$, so we easily get

$$
\mathbb{P}\{\varphi(\gamma(x))=j, M=0, H>0 \mid \varphi(0)=i, X(0)=x\}=\int_{0}^{x} e^{Q_{--y}} Q_{-+} e^{Q_{++} y} d y
$$

For the second term, which corresponds to the sample path of Figure $2 \mathrm{~b}$, the phase process $\varphi$ starts in phase $i \in S^{-}$with a level $x$. It stays in subset $S^{-}$for a duration $y \geq x$, reaching some state $k \in S^{-}$and thus with a level 0 . Next a transition occurs from state $k \in S^{-}$to state $h \in S^{+}$, with rate $Q_{+-}(k, h)$, and the process $\varphi$ reaches state $j \in S^{+}$at time $x$ without leaving subset $S^{+}$. We thus have

$$
\begin{aligned}
\mathbb{P}\{\varphi(\gamma(x)) & =j, M=0, H=0 \mid \varphi(0)=i, X(0)=x\} \\
& =\int_{x}^{\infty} e^{Q_{--} y} d y Q_{-+} e^{Q_{++} x}=\left(-Q_{--}\right)^{-1} e^{Q_{--} x} Q_{-+} e^{Q_{++} x}
\end{aligned}
$$

We consider now the case where there is at least one peak, i.e. $M>0$. A typical example of such a sample path is shown in Figure 2a. Such a sample path can be broken up into five stages as follows : 
1. The phase process $\varphi$ starts in phase $i \in S^{-}$and reaches some state $k \in S^{-}$ at time $x-M=y$ without leaving subset $S^{-}$. This means that the fluid level decreases from level $x$ to level $x-y$. As seen in the previous section, the corresponding probability is equal to $e^{Q_{--y}}(i, k)$.

2. Starting from phase $k \in S^{-}$and level $x-y$, the fluid process returns for the first time to level $x-y$ in some phase $h \in S^{+}$, without exceeding level $x-y$. The corresponding probability is equal to $\Theta(x-y)$.

3. Since a peak (of height equal to $x-y$ at time $\theta(M)$ ) occurs, this means that a transition of the phase process $\varphi$ occurs from state $h \in S^{+}$to some state $m \in S^{-}$. The corresponding transition rate is equal to $Q_{-+}(h, m)$.

4. Once again, starting from phase $m \in S^{-}$and level $x-y$, the fluid process returns for the first time to level $x-y$ in some phase $v \in S^{+}$without exceeding level $x-y$. By the spatial homogeneity of the process, the corresponding probability is equal to $\Theta(x-y)$.

5. The phase process $\varphi$ starts in phase $v \in S^{+}$and level $x-y$. It reaches level $x$ in state $j$ without leaving subset $S^{+}$, at time $\varphi(\gamma(x))$. This means that the fluid level increases from level $x-y$ to level $x$. As seen in the previous section, the corresponding probability is equal to $e^{Q_{++} y}(v, j)$.

We thus obtain

$$
g_{i, j}(x, y)=
$$

$\sum_{k \in S^{-}} \sum_{h \in S^{+}} \sum_{m \in S^{-}} \sum_{v \in S^{+}} e^{Q_{++} y}(i, k) \Theta_{k, h}(x-y) Q_{+-}(h, m) \Theta_{m, v}(x-y) e^{Q_{++} y}(v, j)$,

that is $g(x, y)=e^{Q_{--} y} \Theta(x-y) Q_{+-} \Theta(x-y) e^{Q_{++} y}$.

The matrices $\Theta(x)$ and $G(x, z)$ have been defined only for $x>0$. Clearly, if we set $x=0$, and thus $z=0$, in these definitions, we obtain the zero matrix. In fact, we write $\Theta(0)$ for $\Theta\left(0^{+}\right)$and $G(0,0)$ for $G\left(0^{+}, 0\right)$ which means that $\Theta(0)=\lim _{x \longrightarrow 0} \Theta(x)=\lim _{x \longrightarrow 0} G(x, 0)=G(0,0)$. This simply means that $x=0$ is not a continuity point of these two functions.

The matrices $G(x, z)$ and $\Theta(x)$ are given by the following corollary.

Corollary 8. For $x \geq 0$ and $0 \leq z \leq x$, we have

$$
\begin{aligned}
G(x, z)= & \int_{0}^{x} e^{Q_{--} y} Q_{-+} e^{Q_{++} y} d y+\left(-Q_{--}\right)^{-1} e^{Q_{--} x} Q_{-+} e^{Q_{++} x} \\
& +\int_{0}^{z} e^{Q_{--}} \Theta(x-y) Q_{+-} \Theta(x-y) e^{Q_{++} y} d y \\
\Theta(x)= & \int_{0}^{x} e^{Q_{--} y} Q_{-+} e^{Q_{++} y} d y+\left(-Q_{--}\right)^{-1} e^{Q_{--} x} Q_{-+} e^{Q_{++} x} \\
& +\int_{0}^{x} e^{Q_{--} y} \Theta(x-y) Q_{+-} \Theta(x-y) e^{Q_{++} y} d y
\end{aligned}
$$


Proof. It suffices to write

$$
G(x, z)=G(x, 0)+\int_{0}^{z} g(x, y) d y
$$

and $\Theta(x)=G(x, x)$.

The following result shows that $\Theta(x)$ satisfies a matrix differential Riccati equation.

Theorem 9. $\Theta(0)=\left(-Q_{--}\right)^{-1} Q_{-+}$and, for $x>0$, we have

$$
\Theta^{\prime}(x)=Q_{--} \Theta(x)+\Theta(x) Q_{++}+\Theta(x) Q_{+-} \Theta(x)+Q_{-+} .
$$

Proof. The proof is based on Equation (2.18) and is thus quite similar to the proof of Theorem 5.

Again, the Cauchy-Lipschitz Theorem ensures that the matrix differential Riccati equation (2.19) with the initial condition $\Theta(0)=\left(-Q_{--}\right)^{-1} Q_{-+}$has a unique solution. The following theorem gives an expression of the solution $\Theta(x)$ to that equation. Note that $\Theta(0)$ is a stochastic matrix.

Theorem 10. For every $x \geq 0$, we have $\Theta(x)=Z(x) Y(x)^{-1}$, where the matrices $Y(x)$ and $Z(x)$ are given by

$$
\left(\begin{array}{c}
Z(x) \\
Y(x)
\end{array}\right)=e^{-C Q x}\left(\begin{array}{c}
\Theta(0) \\
I
\end{array}\right) .
$$

Proof. Let us consider the following linear differential equation

$$
Y^{\prime}(x)=\left(-Q_{++}-Q_{+-} \Theta(x)\right) Y(x) \text { and } Y(0)=I .
$$

The function $\Theta(x)$ being continuous, this linear system has a unique solution which is invertible. We now define the $n_{-} \times n_{+}$matrix $Z(x)$ by $Z(x)=$ $\Theta(x) Y(x)$. Using this definition, equation (2.21) becomes

$$
Y^{\prime}(x)=-Q_{++} Y(x)-Q_{+-} Z(x) .
$$

Differentiating $Z(x)$ with respect to $x$, we obtain from (2.19) and (2.22)

$$
\begin{aligned}
Z^{\prime}(x)= & \Theta^{\prime}(x) Y(x)+\Theta(x) Y^{\prime}(x) \\
= & \left(Q_{--} \Theta(x)+\Theta(x) Q_{++}+\Theta(x) Q_{+-} \Theta(x)+Q_{-+}\right) Y(x) \\
& +\Theta(x)\left(-Q_{++} Y(x)-Q_{+-} Z(x)\right) \\
= & Q_{--} Z(x)+\Theta(x) Q_{+-} \Theta(x) Y(x)+Q_{-+} Y(x)-\Theta(x) Q_{+-} Z(x) \\
= & Q_{--} Z(x)+Q_{-+} Y(x) .
\end{aligned}
$$

Putting together equations (2.22) and (2.23) we obtain

$$
\left(\begin{array}{c}
Z^{\prime}(x) \\
Y^{\prime}(x)
\end{array}\right)=\left(\begin{array}{cc}
Q_{--} & Q_{-+} \\
-Q_{+-} & -Q_{++}
\end{array}\right)\left(\begin{array}{c}
Z(x) \\
Y(x)
\end{array}\right)=-C Q\left(\begin{array}{c}
Z(x) \\
Y(x)
\end{array}\right),
$$


with $Y(0)=I$ and $Z(0)=\Theta(0)=\left(-Q_{--}\right)^{-1} Q_{-+}$. The solution to that equation is given by

$$
\left(\begin{array}{c}
Z(x) \\
Y(x)
\end{array}\right)=e^{-C Q x}\left(\begin{array}{c}
\Theta(0) \\
I
\end{array}\right)
$$

which completes the proof.

Note also that Relation (2.20) can also be written as

$$
\left(\begin{array}{c}
Z(x) \\
Y(x)
\end{array}\right)=\left(\begin{array}{cc}
A(x) & B(x) \\
C(x) & D(x)
\end{array}\right)^{-1}\left(\begin{array}{c}
\Theta(0) \\
I
\end{array}\right) .
$$

\section{$3 \quad$ Fluid queue with finite buffer}

We suppose now that the fluid queue is of finite capacity and we denote that capacity by $x$.

\subsection{Congestion metrics}

Assume that the buffer is initially empty $(X(0)=0)$ and that $\varphi(0) \in S^{+}$, so that a busy period starts at time 0 .

We characterize congestion by the distribution of the two random variables :

- the total time $\tau(x)$ during which the buffer is full in a busy period,

- the total quantity of fluid $V(x)$ lost in a busy period.

The random variables $\tau(x)$ and $V(x)$ are formally defined by

$$
\tau(x)=\int_{0}^{\theta(0)} 1_{\{X(t)=x\}} d t \quad \text { and } \quad V(x)=\int_{0}^{\theta(0)} r_{\varphi(t)} 1_{\{X(t)=x\}} d t .
$$

Note that $X(t)=x$ implies that $\varphi(t) \in S^{+} \cup S^{0}$ and when $\varphi(t) \in S^{0}, r_{\varphi(t)}=0$.

We consider the successive periods when the buffer capacity $x$ is reached by process $(X(t))$. We denote by $K$ the number of such periods occurring during a busy period of the buffer. Clearly, $K$ is an integer valued random variable and if $K=0$, which means that process never reaches level $x$ during the busy period, then we have $V(x)=0$. We thus consider the case when $K \geq 1$.

For every $k \geq 1$, we denote by $\gamma_{k}$ the $k$-th instant at which $X\left(\gamma_{k}\right)=x$ and $X\left(\gamma_{k}^{-}\right)<x$. We also denote by $\tau_{k}(x)$ the duration of the $k$-th period during which the fluid level is equal to $x$. More precisely, if we set $\gamma_{0}=0$ and $\tau_{0}(x)=0$, we have for every $k \geq 1$

$$
\begin{aligned}
\gamma_{k} & =\inf \left\{t \in\left(\gamma_{k-1}+\tau_{k-1}(x), \theta(0)\right) \mid X(t)=x\right\}, \\
\tau_{k}(x) & =\inf \left\{t>\gamma_{k} \mid X(t)<x\right\}-\gamma_{k} .
\end{aligned}
$$

We have $\gamma_{1}=\gamma(x)$. In addition, $\gamma_{k}$ also depends on $x$ but we do not mention this dependence to simplify the notation. When $K \geq 1$, for $k \in\{1, \ldots, K\}$, we 
denote by $V_{k}(x)$ the volume of fluid lost during period $\tau_{k}(x)$. This volume is easily expressed by

$$
V_{k}(x)=\int_{\gamma_{k}}^{\tau_{k}(x)+\gamma_{k}} r_{\varphi(t)} d t
$$

and we have

$$
\tau(x)=\sum_{k=1}^{K} \tau_{k}(x) \text { and } V(x)=\sum_{k=1}^{K} V_{k}(x) .
$$

The above variables are shown in Figure 3 in the case when $K=3$. To simplify the figure, we have chosen the nonzero effective input rates equal to -1 or 1 . We have represented in this figure, above the axis $X(t)=x$, the successive volumes of fluid lost during the busy period.

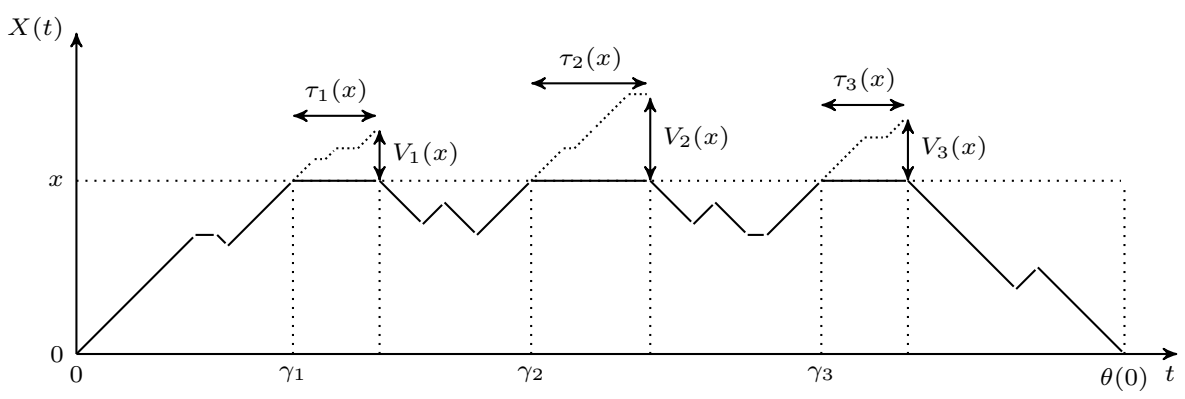

Figure 3: A busy period with losses

Note that, for every $k \geq 1$ and for every $j \in S^{+}$, we have

$$
\varphi\left(\gamma_{k}\right)=j \Longrightarrow \varphi\left(\gamma_{k}\right) \in S^{+} \Longleftrightarrow K \geq k
$$

\subsection{Minimum valley height in a busy period}

We are first interested by the evaluation of the probability, starting from a phase $i \in S^{+}$and a level $z$, to reach level $z$ in phase $j$ while staying above level $z$. This probability is denoted by $\Gamma_{i, j}(x, z)$ which is defined below.

We first introduce the $n_{+} \times n_{-}$matrix $K(x, y, z)$ whose entries are defined, for $i \in S^{+}, j \in S^{-}, x>0$ and $0 \leq z \leq y \leq x$, by

$$
K_{i, j}(x, y, z)=\mathbb{P}\{\varphi(\gamma(z))=j, H \leq y \mid \varphi(0)=i, X(0)=z\},
$$

where $H$ denotes the minimum valley between instants 0 and $\gamma(z)$. When there are no valley, we take as convention $H=0$. This corresponds either to the situation of Figure 4b, i.e. no peak, or to the situation of Figure 4c, i.e. only one peak. 


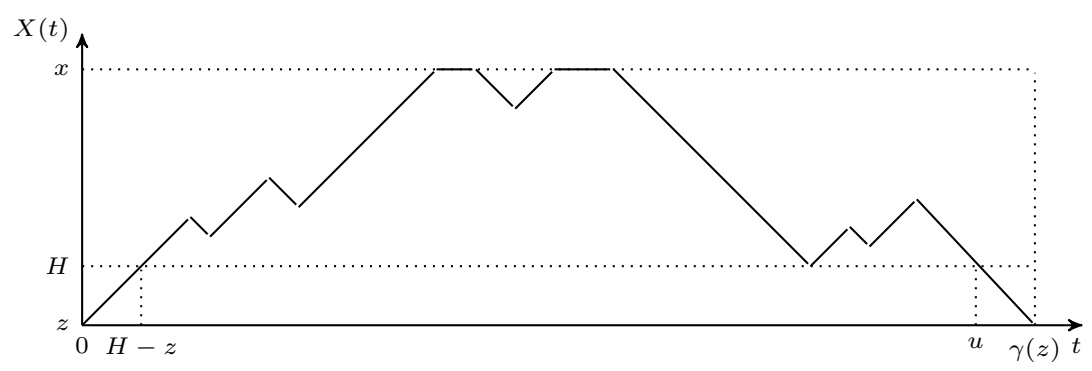

a) with at least 2 peaks $(u=\gamma(z)-H+z)$

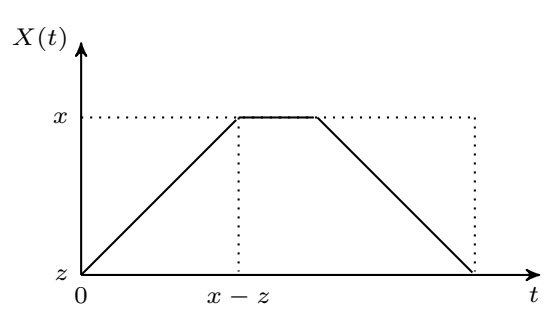

b) without peak

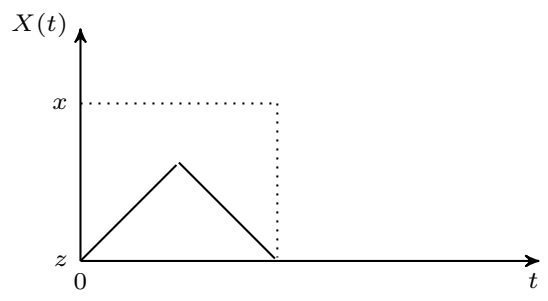

c) with only one peak

Figure 4: A period of level greater than $z$

By the spatial homogeneity, we have $K_{i, j}(x, y, z)=K_{i, j}(x-z, y-z, 0)$. The $n_{+} \times n_{-}$matrix $\Gamma(x, z)$ whose entries are defined, for $i \in S^{+}, j \in S^{-}, x>0$ and $0 \leq z \leq x$, by

$$
\Gamma_{i, j}(x, z)=\mathbb{P}\{\varphi(\gamma(z))=j \mid \varphi(0)=i, X(0)=z\},
$$

satisfies $\Gamma(x, z)=K(x, x, z)=K(x-z, x-z, 0)=\Gamma(x-z, 0)$. The $n_{+} \times n_{-}$ matrix $\Gamma(x)$ whose entries are defined, for $i \in S^{+}, j \in S^{-}$and $x>0$, by

$$
\Gamma_{i, j}(x)=\mathbb{P}\{\varphi(\gamma(0))=j \mid \varphi(0)=i, X(0)=0\},
$$

satisfies $\Gamma(x)=\Gamma(x, 0)$. A typical example of such paths is shown in Figure 4 .

We denote by $k_{i, j}(x, y, z)$ the joint density associated with the joint distribution $K_{i, j}(x, y, z)$, i.e. $k_{i, j}(x, y, z)=\partial K_{i, j}(x, y, z) / \partial y$.

This situation is symmetric to the one studied in Section 2.5 where we have considered the maximum peak between two successive visits to level $x$, starting with a negative net input rate and with a barrier at level 0 . This barrier is natural but it is important to mention it for the comparison with the finite buffer case. Here, we consider the minimum valley between two successive visits to level $z$, starting with a positive net input rate and with a barrier at level $x$. When $z=0$, if we take the symmetry of Figure 3 with respect to the line $X(t)=x / 2$ we obtain a figure similar to Figure 2. Actually, if we exchange the indices + and - of the submatrices of $Q$, in the expression of $g(x, y)$ and $\Theta(x)$ we obtain respectively, as we shall see, $k(x, y, 0)$ and $\Gamma(x)$. 
Theorem 11. For $x>0$ and $0 \leq z \leq y \leq x$, we have

$$
\begin{aligned}
K(x, z, z)= & \int_{z}^{x} e^{Q_{++}(y-z)} Q_{+-} e^{Q_{--}(y-z)} d y \\
& +\left(-Q_{++}\right)^{-1} e^{Q_{++}(x-z)} Q_{+-} e^{Q_{--}(x-z)}, \\
k(x, y, z)= & e^{Q_{++}(y-z)} \Gamma(x-y) Q_{-+} \Gamma(x-y) e^{Q_{--}(y-z)} .
\end{aligned}
$$

Proof. The proof is quasi-identical to the proof of Theorem 7 .

Using the spatial homogeneity, the matrices $K(x, y, z)$ and $\Gamma(x)$ are given by the following equations. For $x \geq 0$ and $0 \leq z \leq y \leq x$, we have

$$
K(x, y, z)=K(x, z, z)+\int_{z}^{y} k(x, u, z) d u .
$$

For $x \geq 0$, we have $\Gamma(x)=\Gamma(x, 0)=K(x, x, 0)$, that is

$$
\begin{aligned}
\Gamma(x)= & \int_{0}^{x} e^{Q_{++} y} Q_{+-} e^{Q_{--} y} d y+\left(-Q_{++}\right)^{-1} e^{Q_{++} x} Q_{+-} e^{Q_{--}} \\
& +\int_{0}^{x} e^{Q_{++} y} \Gamma(x-y) Q_{-+} \Gamma(x-y) e^{Q_{--} y} d y .
\end{aligned}
$$

This equation is the symmetric version of equation (2.18). We thus obtain, in the same way we got for equation (2.18), the matrix differential Riccati equation

$$
\Gamma^{\prime}(x)=Q_{++} \Gamma(x)+\Gamma(x) Q_{--}+\Gamma(x) Q_{-+} \Gamma(x)+Q_{+-} .
$$

This equation is identical to equation (2.10). The only difference concerns the initial condition. Here we have, from $(3.5), \Gamma(0)=\left(-Q_{++}\right)^{-1} Q_{+-}$. Following the same lines used in the proof of Theorem 6 , we obtain $\Gamma(x)=Z(x) Y^{-1}(x)$, where

$$
\left(\begin{array}{c}
Y(x) \\
Z(x)
\end{array}\right)=e^{C Q x}\left(\begin{array}{c}
I \\
\Gamma(0)
\end{array}\right)
$$

Note that $\Gamma(0)=\left(-Q_{++}\right)^{-1} Q_{+-}$is a stochastic matrix. This can be written as

$$
\left\{\begin{array}{l}
Y(x)=A(x)+B(x) \Gamma(0) \\
Z(x)=C(x)+D(x) \Gamma(0)
\end{array}\right.
$$

which gives

$$
\Gamma(x)=[C(x)+D(x) \Gamma(0)][A(x)+B(x) \Gamma(0)]^{-1} .
$$

\subsection{Reduction of the state space}

As we did in Section 2.2, we introduce the $\left(n_{-}+n_{+}\right) \times\left(n_{-}+n_{+}\right)$matrix

$$
Q=\left(\begin{array}{ll}
Q_{--} & Q_{-+} \\
Q_{+-} & Q_{++}
\end{array}\right),
$$


where $Q_{--}=R_{-}^{-1}\left(T_{--}-T_{-0} T_{00}^{-1} T_{0-}\right), Q_{-+}=R_{-}^{-1}\left(T_{-+}-T_{-0} T_{00}^{-1} T_{0+}\right)$, $Q_{+-}=R_{+}^{-1}\left(T_{+-}-T_{+0} T_{00}^{-1} T_{0-}\right)$ and $Q_{++}=R_{+}^{-1}\left(T_{++}-T_{+0} T_{00}^{-1} T_{0+}\right)$. As shown in [4] and [19], when considering quantities such as hitting probabilities and accumulated rewards during sojourn times in $S^{+} \cup S^{0}$, the fluid queue with parameters $(T, R)$ is equivalent to the fluid queue with parameters $(Q, C)$ where $C$ is the $\left(n_{-}+n_{+}\right) \times\left(n_{-}+n_{+}\right)$matrix

$$
C=\left(\begin{array}{cc}
-I & 0 \\
0 & I
\end{array}\right) \text {. }
$$

The distribution of $\tau_{k}(x)$, given that the phase at time $\gamma_{k}$ is $j \in S^{+}$, is given by

$$
\mathbb{P}\left\{\tau_{k}(x)>t \mid \varphi\left(\gamma_{k}\right)=j\right\}=\left(e^{A t} \mathbb{1}\right)_{j},
$$

where $A$ is the $\left(n_{+}+n_{0}\right) \times\left(n_{+}+n_{0}\right)$ matrix defined by

$$
A=\left(\begin{array}{cc}
T_{00} & T_{0+} \\
T_{+0} & T_{++}
\end{array}\right)
$$

and $\mathbb{1}$ is the column vector with all its entries equal to 1 , its dimension being defined by the context. Since $j \in S^{+}$, we only need the entries of $e^{A t} \mathbb{1}$ corresponding to the subset $S^{+}$, so we decompose vector $e^{A t} \mathbb{1}$ with respect to subsets $S^{0}$ and $S^{+}$, by writing

$$
e^{A t} \mathbb{1}=\left(\begin{array}{c}
\left(e^{A t} \mathbb{1}\right)^{0} \\
\left(e^{A t} \mathbb{1}\right)^{+}
\end{array}\right) .
$$

We then have

$$
\mathbb{P}\left\{\tau_{k}(x)>t \mid \varphi\left(\gamma_{k}\right)=j\right\}=\left(e^{A t} \mathbb{1}\right)_{j}^{+} .
$$

Now, if we consider the volume of fluid $V_{k}(x)$ lost during period $\tau_{k}(x)$, given that the phase at time $\gamma_{k}$ is $j, V_{k}(x)$ can also be seen as the accumulated reward during a sojourn of Markov chain $(\varphi(t))$ in subset $S^{0} \cup S^{+}$, starting in state $j \in S^{+}$. Using the results of [19], we get, for $j \in S^{+}$,

$$
\mathbb{P}\left\{V_{k}(x)>v \mid \varphi\left(\gamma_{k}\right)=j\right\}=\left(e^{Q_{++}} \mathbb{1}\right)_{j} .
$$

Thus, in the following, we will consider a fluid queue driven by a Markov chain $(\varphi(t))$ with state space $S=S^{-} \cup S^{+}$, infinitesimal generator $Q$ and effective input rates given by matrix $C$, i.e. equal to -1 or 1 .

\subsection{Distributions of $\tau_{1}(x)$ and $V_{1}(x)$}

\subsubsection{Matrix differential equation}

In order to determine the distribution of $\tau_{1}(x)$ and $V_{1}(x)$, we need the distribution of the Markov chain $(\varphi(t))$ at time $\gamma_{1}$. We introduce, for $i \in S^{+}$and $j \in S^{+}$,

$$
M_{i, j}(x)=\mathbb{P}\left\{\varphi\left(\gamma_{1}\right)=j \mid \varphi(0)=i, X(0)=0\right\}
$$


and we denote by $M(x)$ the matrix with entries $M_{i, j}(x)$. By definition of matrix $\Psi(x)$, which has already been defined in the infinite buffer case by Relation (2.7), for all $i \in S^{+}$, the quantity $\sum_{j \in S^{-}} \Psi_{i, j}(x)$ is the probability that the process does not hit level $x$ in a busy period starting from state $i$ and an empty buffer and $\sum_{j \in S^{+}} M_{i, j}(x)$ is the probability of the complementary event. Hence, for all $i \in S^{+}$, we have $\sum_{j \in S^{-}} \Psi_{i, j}(x)+\sum_{j \in S^{+}} M_{i, j}(x)=1$. This can be written in matrix form as $\Psi(x) \mathbb{1}+M(x) \mathbb{1}=\mathbb{1}$, where we recall that the dimension of $\mathbb{1}$ is given by the context of its use.

Matrices $M(x)$ and $\Psi(x)$ are related one to each other via a differential and an integral equation. As shown in Theorem 4 and Theorem 5, Matrix $\Psi(x)$ satisfies the integral equation (2.9) and the matrix differential Riccati equation (2.10). Matrix $M(x)$ is then related to $\Psi(x)$ by the following result.

Theorem 12. For every $x \geq 0$, we have

$$
\begin{gathered}
M(x)=e^{Q_{++} x}+\int_{0}^{x} e^{Q_{++} y} \Psi(x-y) Q_{-+} M(x-y) d y \\
M^{\prime}(x)=\left(Q_{++}+\Psi(x) Q_{-+}\right) M(x) .
\end{gathered}
$$

Proof. The proof of (3.10) follows the arguments developed in the proof of Theorem 4 and (3.11) is obtained by differentiating (3.10).

\subsubsection{Distributions of the random variables}

The distributions of $\tau_{1}(x)$ and $V_{1}(x)$ are given by the following theorem.

Theorem 13. For every $i \in S^{+}$and for all $v, t \geq 0$, we have

$$
\begin{gathered}
\mathbb{P}\left\{\tau_{1}(x)>t \mid \varphi(0)=i\right\}=\left(M(x)\left(e^{A t} \mathbb{1}\right)^{+}\right)_{i}, \\
\mathbb{P}\left\{V_{1}(x)>v \mid \varphi(0)=i\right\}=\left(M(x) e^{Q_{++}{ }^{v}} \mathbb{1}\right)_{i}, \\
\mathbb{P}\{K \geq 1 \mid \varphi(0)=i\}=(M(x) \mathbb{1})_{i} .
\end{gathered}
$$

Proof. Using the Markov property, we obtain, for every $i \in S^{+}$,

$$
\begin{aligned}
\mathbb{P}\left\{\tau_{1}(x)>t \mid \varphi(0)=i\right\} & =\sum_{j \in S^{+}} \mathbb{P}\left\{\tau_{1}(x)>t \mid \varphi\left(\gamma_{1}\right)=j\right\} \mathbb{P}\left\{\varphi\left(\gamma_{1}\right)=j \mid \varphi(0)=i\right\} \\
& =\sum_{j \in S^{+}} M_{i, j}(x)\left(e^{A t} \mathbb{1}\right)_{j}^{+}=\left(M(x)\left(e^{A t} \mathbb{1}\right)^{+}\right)_{i} .
\end{aligned}
$$

The same argument leads to Relation (3.13). From Equivalence (3.1), we get, by definition of $M_{i, j}(x), \mathbb{P}\{K \geq 1 \mid \varphi(0)=i\}=\mathbb{P}\left\{\varphi\left(\gamma_{1}\right) \in S^{+} \mid \varphi(0)=i\right\}=$ $(M(x) \mathbb{1})_{i}$.

In the next subsection, we study the distributions of the state initiating the successive idle and busy periods of the buffer. 


\subsection{Sequences of idle and busy periods}

We decompose the initial probability distribution $\alpha$ of Markov chain $(\varphi(t))$ through the partition $S^{-}, S^{0}, S^{+}$, by writing $\alpha=\left(\alpha^{-}, \alpha^{0}, \alpha^{+}\right)$. Recall that we suppose that the buffer is initially empty, i.e. that $X(0)=0$ with probability 1 . For every $n \geq 1$, we denote by $S I_{n}$ the state or the phase by which the $n$-th idle period starts and we denote by $S B_{n}$ the state or the phase by which the $n$-th busy period starts. We then have $S I_{1} \in S^{-} \cup S^{0}$ if $\alpha^{0} \neq 0, S I_{n} \in S^{-}$, for $n \geq 2$ and $S B_{n} \in S^{+}$. We denote respectively by $v_{n}$ and $w_{n}$ the probability distributions of $S I_{n}$ and $S B_{n}$, i.e.

$$
w_{n}=\left(\mathbb{P}\left\{S B_{n}=j\right\}, j \in S^{+}\right),
$$

$v_{1}=\left(\mathbb{P}\left\{S I_{1}=j\right\}, j \in S^{-} \cup S^{0}\right)$ and, for $n \geq 2, v_{n}=\left(\mathbb{P}\left\{S I_{n}=j\right\}, j \in S^{-}\right)$. Recall that for $i \in S^{+}$and $j \in S^{-}$, the probability $\Gamma_{i, j}(x)$ that a busy period of the buffer with capacity $x$, ends in phase $j$ given that it starts in phase $i$, that is

$$
\Gamma_{i, j}(x)=\mathbb{P}\{\varphi(\theta(0))=j \mid \varphi(0)=i\}
$$

is given by Relation (3.7). Remark that for every $x \geq 0$, we have $\Gamma(x) \mathbb{1}=\mathbb{1}$. Using the results of [19], we have

$$
v_{1}=\left(\alpha^{-}+\alpha^{+} \Gamma(x), \alpha^{0}\right) \text { and } w_{1}=\alpha^{+}+\left(\alpha^{-}, \alpha^{0}\right)\left(-A^{\prime}\right)^{-1} B^{\prime},
$$

where matrices $A^{\prime}$ and $B^{\prime}$ are defined by

$$
A^{\prime}=\left(\begin{array}{cc}
T_{--} & T_{-0} \\
T_{0-} & T_{00}
\end{array}\right) \text { and } B^{\prime}=\left(\begin{array}{c}
T_{-+} \\
T_{0+}
\end{array}\right) .
$$

Defining $Q_{00}=T_{00}-T_{0-} T_{--}^{-1} T_{-0}$ and $Q_{0+}=T_{0+}-T_{0-} T_{--}^{-1} T_{-+}$, we have

$$
\left(-A^{\prime}\right)^{-1}=\left(\begin{array}{cc}
\left(-Q_{--}\right)^{-1} R_{-}^{-1} & \left(-Q_{--}\right)^{-1} R_{-}^{-1} T_{-0}\left(-T_{00}\right)^{-1} \\
\left(-Q_{00}\right)^{-1} T_{0-}\left(-T_{--}\right)^{-1} & \left(-Q_{00}\right)^{-1}
\end{array}\right)
$$

and

$$
\left(-A^{\prime}\right)^{-1} B^{\prime}=\left(\begin{array}{c}
\left(-Q_{--}\right)^{-1} Q_{-+} \\
\left(-Q_{00}\right)^{-1} Q_{0+}
\end{array}\right)
$$

which leads to

$$
w_{1}=\alpha^{+}+\alpha^{-}\left(-Q_{--}\right)^{-1} Q_{-+}+\alpha^{0}\left(-Q_{00}\right)^{-1} Q_{0+} .
$$

In the following theorem, we give the expressions of $v_{n}$ and $w_{n}$, for $n \geq 2$.

Theorem 14. For every $n \geq 2$, we have

$$
\begin{aligned}
& v_{n}=\left(\alpha^{-}+\alpha^{+} \Gamma(x)\right) \mathcal{L}^{n-1}(x)+\alpha^{0}\left(-Q_{00}\right)^{-1} Q_{0+} \Gamma(x) \mathcal{L}^{n-2}(x), \\
& w_{n}=\left(\alpha^{+}+\alpha^{-}\left(-Q_{--}\right)^{-1} Q_{-+}+\alpha^{0}\left(-Q_{00}\right)^{-1} Q_{0+}\right) \mathcal{K}^{n-1}(x),
\end{aligned}
$$

where matrices $\mathcal{L}(x)$ and $\mathcal{K}(x)$ are given by

$$
\mathcal{L}(x)=\left(-Q_{--}\right)^{-1} Q_{-+} \Gamma(x) \text { and } \mathcal{K}(x)=\Gamma(x)\left(-Q_{--}\right)^{-1} Q_{-+} .
$$


Proof. For every $j \in S^{-}$, we have

$$
\mathbb{P}\left\{S I_{2}=j\right\}=\sum_{i \in S^{-} \cup S^{0}} \mathbb{P}\left\{S I_{1}=i\right\}\left[\left(-A^{\prime}\right)^{-1} B^{\prime} \Gamma(x)\right]_{i, j},
$$

which gives, in matrix notation, using (3.17) and (3.15),

$$
\begin{aligned}
v_{2} & =v_{1}\left(-A^{\prime}\right)^{-1} B^{\prime} \Gamma(x) \\
& =\left(\alpha^{-}+\alpha^{+} \Gamma(x)\right)\left(-Q_{--}\right)^{-1} Q_{-+} \Gamma(x)+\alpha^{0}\left(-Q_{00}\right)^{-1} Q_{0+} \Gamma(x) \\
& =\left(\alpha^{-}+\alpha^{+} \Gamma(x)\right) \mathcal{L}(x)+\alpha^{0}\left(-Q_{00}\right)^{-1} Q_{0+} \Gamma(x) .
\end{aligned}
$$

For every $n \geq 3$ and $j \in S^{-}$, we have, by homogeneity,

$$
\mathbb{P}\left\{S I_{n}=j\right\}=\sum_{i \in S^{-}} \mathbb{P}\left\{S I_{n-1}=i\right\}\left[\left(-A^{\prime}\right)^{-1} B^{\prime} \Gamma(x)\right]_{i, j} .
$$

We thus get, in matrix notation, again using (3.17) and (3.15),

$$
v_{n}=v_{n-1}\left(-Q_{--}\right)^{-1} Q_{-+} \Gamma(x)=v_{n-1} \mathcal{L}(x) .
$$

This leads, for $n \geq 2$, to $v_{n}=v_{2} \mathcal{L}^{n-2}(x)$, which is the desired result. In the same way, we have for every $n \geq 2$ and $j \in S^{+}$, using (3.17),

$$
\begin{aligned}
\mathbb{P}\left\{S B_{n}=j\right\} & =\sum_{i \in S^{+}} \mathbb{P}\left\{S B_{n-1}=i\right\} \sum_{\ell \in S^{-}} \Gamma_{i, \ell}(x)\left[\left(-A^{\prime}\right)^{-1} B^{\prime}\right]_{\ell, j} \\
& =\sum_{i \in S^{+}} \mathbb{P}\left\{S B_{n-1}=i\right\}\left[\Gamma(x)\left(-Q_{--}\right)^{-1} Q_{-+}\right]_{i, j} \\
& =\sum_{i \in S^{+}} \mathbb{P}\left\{S B_{n-1}=i\right\} \mathcal{K}_{i, j}(x) .
\end{aligned}
$$

This gives in matrix notation, using (3.18), $w_{n}=w_{n-1} \mathcal{K}(x)$, that is $w_{n}=$ $w_{1} \mathcal{K}^{n-1}(x)$.

The Markov chain $(\varphi(t))$ being finite and irreducible, matrices $\mathcal{L}(x)$ and $\mathcal{K}(x)$ which are stochastic matrices are also irreducible and aperiodic. We thus have

$$
\lim _{n \longrightarrow \infty} v_{n}=\pi^{-} \text {and } \lim _{n \longrightarrow \infty} w_{n}=\pi^{+},
$$

where $\pi^{-}$is a row vector of dimension $n_{-}$which is the unique solution to the system $\pi^{-}=\pi^{-} \mathcal{L}(x)$, with $\pi^{-} \mathbb{1}=1$ and $\pi^{+}$is a row vector of dimension $n_{+}$ which is the unique solution to the system $\pi^{+}=\pi^{+} \mathcal{K}(x)$, with $\pi^{+} \mathbb{1}=1$.

In the sequel, we suppose that the queue is in stationary regime, that is that the distribution of $\varphi(0)$ is $\pi^{+}$. We thus have $\mathbb{P}\left\{\tau_{1}(x)>t\right\}=\pi^{+} M(x)\left(e^{A t} \mathbb{1}\right)^{+}$, $\mathbb{P}\left\{V_{1}(x)>v\right\}=\pi^{+} M(x) e^{Q_{++} v} \mathbb{1}$ and $\mathbb{P}\{K \geq 1\}=\pi^{+} M(x) \mathbb{1}$. The above result gives the distribution of the first congestion event. In the next section, we compute the characteristics of several congestion events. 


\subsection{Joint distributions of loss periods and loss volumes}

In this section, we study the joint distribution of the times $\tau_{k}(x)$ and the joint distribution of the volumes $V_{k}(x)$. To deal with these distributions, we need to evaluate the probability $H_{i, j}(x)$, starting from level $x$ in phase $i \in S^{-}$, that the phase at the time of the first return to level $x$ is equal to $j$, without hitting level 0 . This probability can be interpreted as the symmetric probability of $\Psi(x)$ with respect to levels 0 and $x$. It is defined, for every $i \in S^{-}$and $j \in S^{+}$, by

$$
H_{i, j}(x)=\mathbb{P}\{\varphi(\gamma(x))=j, X(s)>0 \text { for } 0 \leq s \leq \gamma(x) \mid \varphi(0)=i, X(0)=x\} .
$$

Theorem 15. For every $x \geq 0$, we have

$$
H(x)=\int_{0}^{x} e^{Q_{--} y} Q_{-+} e^{Q_{++} y} d y+\int_{0}^{x} e^{Q_{--} y} H(x-y) Q_{+-} H(x-y) e^{Q_{++} y} d y .
$$

Proof. The proof is quite similar to the proof leading to Equation (2.9). It can also be seen as a particular case of the way in which Equation (2.18) has been obtained since here we do not consider the paths hitting level 0 . and

In the same way we obtained Equation (2.10), matrix $H(x)$ satisfies $H(0)=0$

$$
H^{\prime}(x)=Q_{--} H(x)+H(x) Q_{++}+H(x) Q_{+-} H(x)+Q_{-+} .
$$

Note that, by homogeneity of $\varphi$, we also have, for every $i \in S^{-}, j \in S^{+}$and $k \geq 1$,

$$
H_{i, j}(x)=\mathbb{P}\left\{\varphi\left(\gamma_{k+1}\right)=j \mid \varphi\left(\gamma_{k}+\tau_{k}\right)=i\right\} .
$$

This relation is used to prove the next lemma. We introduce the matrix $B$ defined by

$$
B=\left(\begin{array}{c}
T_{+-} \\
T_{0-}
\end{array}\right)
$$

Lemma 16. For every $k \geq 1, v \geq 0$ and $i, j \in S^{+}$, we have

$$
\mathbb{P}\left\{V_{k}(x)>v, \varphi\left(\gamma_{k+1}\right)=j \mid \varphi\left(\gamma_{k}\right)=i\right\}=\left(e^{Q_{++} v}\left(-Q_{++}\right)^{-1} Q_{+-} H(x)\right)_{i, j}
$$

and

$$
\mathbb{P}\left\{\tau_{k}(x)>t, \varphi\left(\gamma_{k+1}\right)=j \mid \varphi\left(\gamma_{k}\right)=i\right\}=\left(\left[e^{A t}(-A)^{-1} B\right]_{+-} H(x)\right)_{i, j},
$$

where matrix $\left[e^{A t}(-A)^{-1} B\right]_{+-}$is the block $n_{+} \times n_{-}$of matrix $e^{A t}(-A)^{-1} B$.

Proof. The homogeneity of Markov chain $\varphi$ implies that, for every $k \geq 1$,

$\mathbb{P}\left\{V_{k}(x)>v, \varphi\left(\gamma_{k+1}\right)=j \mid \varphi\left(\gamma_{k}\right)=i\right\}=\mathbb{P}\left\{V_{1}(x)>v, \varphi\left(\gamma_{2}\right)=j \mid \varphi\left(\gamma_{1}\right)=i\right\}$ 
so we set $k=1$. Note that the entry $(i, \ell)$ of matrix $e^{Q_{++} v}\left(-Q_{++}\right)^{-1} Q_{+-}$is, see [19], the probability, starting from $i \in S^{+}$, that the accumulated reward during a sojourn of $\varphi$ in $S^{+} \cup S^{0}$ is greater than $v$ and that the first state of $S^{-}$visited after this sojourn is $\ell$. Conditioning on the phase at time $\gamma_{1}+\tau_{1}$ and using the Markov property, we get

$$
\begin{aligned}
& \mathbb{P}\left\{\varphi\left(\gamma_{2}\right)=j, V_{1}(x)>v \mid \varphi\left(\gamma_{1}\right)=i\right\} \\
& =\sum_{\ell \in S^{-}} \mathbb{P}\left\{\varphi\left(\gamma_{2}\right)=j \mid \varphi\left(\gamma_{1}+\tau_{1}\right)=\ell\right\} \mathbb{P}\left\{\varphi\left(\gamma_{1}+\tau_{1}\right)=\ell, V_{1}(x)>v \mid \varphi\left(\gamma_{1}\right)=i\right\} \\
& =\sum_{\ell \in S^{-}} H_{\ell, j}\left(e^{Q_{++} v}\left(-Q_{++}\right)^{-1} Q_{+-}\right)_{i, \ell}=\left(e^{Q_{++} v}\left(-Q_{++}\right)^{-1} Q_{+-} H(x)\right)_{i, j} .
\end{aligned}
$$

To get the second relation, we proceed in the same way noting that the entry $(i, \ell)$ of matrix $\left[e^{A t}(-A)^{-1} B\right]_{+-}$is, see [19], the probability, starting from $i \in$ $S^{+}$, that a sojourn of $\varphi$ in $S^{+} \cup S^{0}$ is greater than $t$ and that the first state of $S^{-}$visited after this sojourn is $\ell$.

Remark that we have the following relations:

$$
\left[(-A)^{-1} B\right]_{+-}=\left(-Q_{++}\right)^{-1} Q_{+-} \text {and }\left(-Q_{++}\right)^{-1} Q_{+-} \mathbb{1}=\mathbb{1} \text {. }
$$

Theorem 17. For every $k \geq 1$ and $v_{1}, \ldots, v_{k} \geq 0$, we have

$$
\begin{aligned}
\mathbb{P}\left\{V_{1}(x)>v_{1}, V_{2}(x)>v_{2}, \ldots, V_{k}(x)>v_{k}\right\} & \\
= & \pi^{+} M(x)\left(\prod_{\ell=1}^{k-1} e^{Q_{++} v_{\ell}}\left(-Q_{++}\right)^{-1} Q_{+-} H(x)\right) e^{Q_{++} v_{k}} \mathbb{1} .
\end{aligned}
$$

Proof. Note that by convention the product is equal to 1 for $k=1$. Let us define $F_{i, j}(k)$, for every $k \geq 1$ and $i, j \in S^{+}$, by

$$
F_{i, j}(k)=\mathbb{P}\left\{V_{1}(x)>v_{1}, \ldots, V_{k}(x)>v_{k}, \varphi\left(\gamma_{k+1}\right)=j \mid \varphi(0)=i\right\} .
$$

Conditioning on $\varphi\left(\gamma_{k}\right)$ and using the Markov property, we have

$$
\begin{aligned}
F_{i, j}(k) & =\sum_{\ell \in S^{+}} \mathbb{P}\left\{V_{1}(x)>v_{1}, \ldots, V_{k}(x)>v_{k}, \varphi\left(\gamma_{k+1}\right)=j, \varphi\left(\gamma_{k}\right)=\ell \mid \varphi(0)=i\right\} \\
& =\sum_{\ell \in S^{+}} F_{i, \ell}(k-1) \mathbb{P}\left\{V_{k}(x)>v_{k}, \varphi\left(\gamma_{k+1}\right)=j \mid \varphi\left(\gamma_{k}\right)=\ell\right\}
\end{aligned}
$$

Denoting by $F(k)$ the matrix $\left[F_{i, j}(k)\right]_{i, j \in S^{+}}$, we obtain, using Lemma 16 ,

$$
F(k)=F(k-1) e^{Q_{++} v_{k}}\left(-Q_{++}\right)^{-1} Q_{+-} H(x),
$$

and thus,

$$
F(k)=F(1) \prod_{\ell=2}^{k} e^{Q_{++} v_{\ell}}\left(-Q_{++}\right)^{-1} Q_{+-} H(x) .
$$


Matrix $F(1)$ is obtained in the same way, using the Markov property and Lemma 16, as

$$
\begin{aligned}
F_{i, j}(1) & =\sum_{\ell \in S^{+}} \mathbb{P}\left\{V_{1}(x)>v_{1}, \varphi\left(\gamma_{2}\right)=j, \varphi\left(\gamma_{1}\right)=\ell \mid \varphi(0)=i\right\} \\
& =\sum_{\ell \in S^{+}} \mathbb{P}\left\{\varphi\left(\gamma_{1}\right)=\ell \mid \varphi(0)=i\right\} \mathbb{P}\left\{V_{1}(x)>v_{1}, \varphi\left(\gamma_{2}\right)=j \mid \varphi\left(\gamma_{1}\right)=\ell\right\} \\
& =\sum_{\ell \in S^{+}} M_{i, \ell}(x)\left[e^{Q_{++} v_{1}}\left(-Q_{++}\right)^{-1} Q_{+-} H(x)\right]_{\ell, j} .
\end{aligned}
$$

Finally, we have

$$
F(k)=M(x) \prod_{\ell=1}^{k} e^{Q_{++} v_{\ell}}\left(-Q_{++}\right)^{-1} Q_{+-} H(x) .
$$

To obtain the joint distribution of $V_{1}(x), \ldots, V_{k}(x)$, we use Relation (3.9), the previous relation and the Markov property, by writing

$$
\begin{aligned}
\mathbb{P}\{ & \left.V_{1}(x)>v_{1}, V_{2}(x)>v_{2}, \ldots, V_{k}(x)>v_{k} \mid \varphi(0)=i\right\} \\
& =\sum_{j \in S^{+}} \mathbb{P}\left\{V_{1}(x)>v_{1}, \ldots, V_{k-1}(x)>v_{k-1}, \varphi\left(\gamma_{k}\right)=j, V_{k}(x)>v_{k} \mid \varphi(0)=i\right\} \\
& =\sum_{j \in S^{+}} F_{i, j}(k-1) \mathbb{P}\left\{V_{k}(x)>v_{k} \mid \varphi\left(\gamma_{k}\right)=j\right\} \\
& =\sum_{j \in S^{+}} F_{i, j}(k-1)\left(e^{Q_{++} v_{k}} \mathbb{1}\right)_{j}=\left[F(k-1) e^{Q_{++} v_{k}} \mathbb{1}\right]_{i} .
\end{aligned}
$$

Since the distribution of $\varphi(0)$ is $\pi^{+}$, we get by unconditioning

$$
\mathbb{P}\left\{V_{1}(x)>v_{1}, V_{2}(x)>v_{2}, \ldots, V_{k}(x)>v_{k}\right\}=\pi^{+} F(k-1) e^{Q_{++} v_{k}} \mathbb{1},
$$

which completes the proof.

Similar arguments are used to get the joint distribution of the $\tau_{k}(x)$.

Theorem 18. For every $k \geq 1$ and $t_{1}, \ldots, t_{k} \geq 0$, we have

$$
\begin{aligned}
\mathbb{P}\left\{\tau_{1}(x)>t_{1}, \tau_{2}(x)>t_{2}, \ldots, \tau_{k}(x)>t_{k}\right\} & \\
& =\pi^{+} M(x)\left(\prod_{\ell=1}^{k-1}\left(e^{A t_{\ell}}(-A)^{-1} B\right)_{+-} H(x)\right)\left(e^{A t_{k}} \mathbb{1}\right)^{+} .
\end{aligned}
$$

Note that the joint distribution of the $\tau_{k}$, given by (3.24), can be rewritten as

$$
\mathbb{P}\left\{\tau_{1}(x)>t_{1}, \ldots, \tau_{k}(x)>t_{k}\right\}=a(x)\left(\prod_{\ell=1}^{k-1} e^{A t_{\ell}}(-A)^{-1} B R(x)\right) e^{A t_{k}} \mathbb{1},
$$


where $a(x)$ is the row vector defined by $a(x)=\left(\pi^{+} M(x) 0\right), 0$ being here the null row vector of dimension $n_{0}$, and $R(x)$ is the matrix defined by $R(x)=(H(x) 0)$, 0 being here the $n_{-} \times n_{0}$ null matrix.

Corollary 19. For every $k \geq 1$ and $v, t \geq 0$, we have

$$
\begin{aligned}
& \mathbb{P}\left\{V_{k}(x)>v\right\}=\pi_{+} M(x)\left(\left(-Q_{++}\right)^{-1} Q_{+-} H(x)\right)^{k-1} e^{Q_{++} v} \mathbb{1}, \\
& \mathbb{P}\left\{\tau_{k}(x)>t\right\}=\pi_{+} M(x)\left(\left(-Q_{++}\right)^{-1} Q_{+-} H(x)\right)^{k-1}\left(e^{A t} \mathbb{1}\right)^{+} .
\end{aligned}
$$

Proof. To get Relation (3.26), put $v_{1}=\cdots=v_{k-1}=0$ and $v_{k}=v$ in (3.23). To get Relation (3.27), put $t_{1}=\cdots=t_{k-1}=0$ and $t_{k}=t$ in (3.24) and observe that $\left((-A)^{-1} B\right)_{+-}=\left(-Q_{++}\right)^{-1} Q_{+-}$.

Corollary 20. For every $k \geq 0$, we have

$$
\mathbb{P}\{K>k\}=\pi^{+} M(x)\left(\left(-Q_{++}\right)^{-1} Q_{+-} H(x)\right)^{k} \mathbb{1} .
$$

Proof. For every $k \geq 0$, we have $V_{k+1}(x)>0 \Longleftrightarrow \tau_{k+1}(x)>0 \Longleftrightarrow K>k$. We thus get the result using either (3.26) or (3.27).

As we did for the joint distribution of the $\tau_{k}$, obtained in (3.25), the distribution of $K$, given by (3.28), can be rewritten as

$$
\mathbb{P}\{K>k\}=a(x)\left((-A)^{-1} B R(x)\right) \mathbb{1} .
$$

\subsection{Total duration of losses and volume of information lost}

We determine in this section the distribution of the total loss duration $\tau(x)$ in a busy period and the distribution of the total volume of information lost $V(x)$ in a busy period. We have seen in Section 3.1 that we have

$$
\tau(x)=\sum_{k=1}^{K} \tau_{k}(x) \text { and } V(x)=\sum_{k=1}^{K} V_{k}(x) .
$$

To get the distribution of $\tau(x)$ and $V(x)$, we need some results about absorbing Markovian arrival processes.

\subsubsection{Absorbing Markovian arrival process}

The Markovian arrival process is well-known in the queuing literature and were introduced in [13]. We consider here a variant of this process in which we add an absorbing state. This process is defined as a continuous-time Markov chain $\{(N(t), J(t)), t \geq 0\}$, with $N(0)=0$, on the state space $S=\{(a, 0)\} \cup$ 
$(\mathbb{N} \times\{1, \ldots, m\})$, with $m \geq 1$ and where state $(a, 0)$ is absorbing. Its infinitesimal generator $G$ is given by

$$
G=\left(\begin{array}{cccccccc}
0 & 0 & 0 & 0 & 0 & 0 & 0 & \ldots \\
V & D_{0} & D_{1} & 0 & 0 & 0 & 0 & \ldots \\
V & 0 & D_{0} & D_{1} & 0 & 0 & 0 & \ldots \\
V & 0 & 0 & D_{0} & D_{1} & 0 & 0 & \ldots \\
\vdots & \vdots & \vdots & \vdots & \vdots & \ddots & \ddots & \ddots
\end{array}\right)
$$

where the first row and the first column correspond to the absorbing state $a$. Matrices $D_{0}$ and $D_{1}$ are non zero $(m, m)$ matrices and column vector $V$ is a non zero vector with dimension $m$. The matrices $G$ being an infinitesimal generator, we have $D_{0} \mathbb{1}+D_{1} \mathbb{1}+V=0$. We moreover assume that all the states, except state $(a, 0)$, are transient. This implies in particular that matrix $D_{0}$ is invertible. For $\ell \geq 0$, we define the subset of states $S_{\ell}=\{(\ell, 1), \ldots,(\ell, m)\}$. As in [22], we set $\xi_{0}=0$ and we denote, for all $n \geq 1$, by $\xi_{n}$ the instant at which either the $n$-th arrival occurs or the absorbing state is reached. Variable $\xi_{n}$ is then defined, for all $n \geq 0$, by $\xi_{n}=\inf \{t \geq 0 \mid N(t) \in\{n, a\}\}$. The $n$-th inter-arrival time $\Gamma_{n}$ is then defined, for $n \geq 1$, by $\Gamma_{n}=\xi_{n}-\xi_{n-1}$. We also define $Y$ as the number of subsets $S_{\ell}$ visited by the process until absorption, i.e.

$$
Y=\sum_{\ell=1}^{\infty} \mathbb{1}_{\left\{\Gamma_{\ell}>0\right\}}
$$

We then have $\xi_{n}=\xi_{Y}$ for $n \geq Y$ and thus $\Gamma_{Y}>0$ and $\Gamma_{n}=0$ for $n \geq Y+1$. Since $N(0)=0$, the initial probability distribution is concentrated on the states $(0,1), \ldots,(0, m)$. We denote by $\beta$ the row vector of dimension $m$ defined by $\beta_{i}=\mathbb{P}\{J(0)=i\}$, for $i=1, \ldots, m$. We thus have $\beta_{1}+\cdots+\beta_{m}=1$. The joint distribution of $\Gamma_{1}, \ldots, \Gamma_{k}$ is given, following the results of [22], by

$$
\mathbb{P}\left\{\Gamma_{1}>t_{1}, \ldots, \Gamma_{k}>t_{k}\right\}=\beta\left[\prod_{\ell=1}^{k-1} e^{D_{0} t_{\ell}}\left(-D_{0}\right)^{-1} D_{1}\right] e^{D_{0} t_{k}} \mathbb{1} .
$$

We then have, for every $k \geq 0, \Gamma_{k+1}>0 \Longleftrightarrow Y>k$. By taking $t_{1}=\cdots=$ $t_{k-1}=0$ and $t_{k}=t$ in (3.30), we get, for every $k \geq 1$ and $t \geq 0$,

$$
\mathbb{P}\left\{\Gamma_{k}>t\right\}=\beta\left(\left(-D_{0}\right)^{-1} D_{1}\right)^{k-1} e^{D_{0} t} \mathbb{1}
$$

that is

$$
\mathbb{P}\{Y>k\}=\beta\left(\left(-D_{0}\right)^{-1} D_{1}\right)^{k} \mathbb{1} .
$$

We denote by $\Gamma$ the total time spent by the process in the transient states, that is $\Gamma=\inf \{t \geq 0 \mid N(t)=a\}$. By definition of $Y$, we have

$$
\Gamma=\sum_{k=1}^{Y} \Gamma_{k}=\sum_{k=1}^{\infty} \Gamma_{k}
$$


The distribution of $\Gamma$ is given, for every $t \geq 0$, by $\mathbb{P}\{\Gamma>t\}=(\beta, 0,0, \ldots) e^{L t} \mathbb{1}$, where

$$
L=\left(\begin{array}{ccccccc}
D_{0} & D_{1} & 0 & 0 & 0 & 0 & \ldots \\
0 & D_{0} & D_{1} & 0 & 0 & 0 & \ldots \\
0 & 0 & D_{0} & D_{1} & 0 & 0 & \cdots \\
\vdots & \vdots & \vdots & \vdots & \ddots & \ddots & \ddots
\end{array}\right)
$$

It is easily checked that for every $k \geq 0$, we have

$$
L^{k} \mathbb{1}=\left(\begin{array}{c}
\left(D_{0}+D_{1}\right)^{k} \mathbb{1} \\
\left(D_{0}+D_{1}\right)^{k} \mathbb{1} \\
\vdots
\end{array}\right)
$$

We then get

$$
\mathbb{P}\{\Gamma>t\}=\beta e^{\left(D_{0}+D_{1}\right) t} \mathbb{1}
$$

\subsubsection{Distribution of $\tau(x)$ and $V(x)$}

Using the notation $a(x)$ and $R(x)$ introduced in (3.25), we obtain the following result.

Theorem 21. For every $v, t \geq 0$, we have

$$
\begin{gathered}
\mathbb{P}\{V(x)>v\}=\pi^{+} M(x) e^{\left(Q_{++}+Q_{+-} H(x)\right) v} \mathbb{1}, \\
\mathbb{P}\{\tau(x)>t\}=a(x) e^{(A+B R(x)) t} \mathbb{1} .
\end{gathered}
$$

Proof. The joint distribution of the $V_{k}$, which is given by (3.23) is equal to the joint distribution of the $\Gamma_{k}$ given by (3.30) by taking $\beta=\pi^{+} M(x), D_{0}=Q_{++}$ and $D_{1}=Q_{+-} H(x)$. With these values, the distribution of $K$, given by (3.28), and the distribution of $Y$, given by (3.31), are identical. We thus have, according to $(3.32)$,

$$
\mathbb{P}\{V(x)>v\}=\pi^{+} M(x) e^{\left(Q_{++}+Q_{+-} H(x)\right) v} \mathbb{1} .
$$

The joint distribution of the $\tau_{k}$ given by (3.25) is equal to the joint distribution of the $\Gamma_{k}$ given by (3.30) by taking $\beta=a(x), D_{0}=A$ and $D_{1}=B R(x)$. With these values, the distribution of $K$, given by (3.29), and the distribution of $Y$, given by (3.31), are identical. We thus have, according to (3.32), $\mathbb{P}\{\tau(x)>t\}=$ $a(x) e^{(A+B R(x)) t} \mathbb{1}$.

\subsubsection{Loss probability}

Let $\mathbb{E}(I P)$ and $\mathbb{E}(B P)$ be the mean durations of an idle and a busy period respectively, in stationary regime and recall that $p$ is the stationary distribution of Markov chain $(\varphi(t))$. The mean input rate in stationary regime is then $\mathbb{E}(\chi)=\sum_{i \in S} p_{i} \chi_{i}$, where we recall that $\chi_{i}$ is the input rate in the queue when 
the modulating Markov chain $(\varphi(t))$ is in state $i$. The quantity $\pi_{\text {loss }}$ defined by Equation (1.1) is then given by

$$
\pi_{\text {loss }}=\frac{1}{\mathbb{E}(I P)+\mathbb{E}(B P)} \frac{\mathbb{E}(V(x))}{\mathbb{E}(\chi)} .
$$

From Relation (3.33), we have

$$
\mathbb{E}(V(x))=\pi^{+} M(x)\left(-Q_{++}-Q_{+-} H(x)\right)^{-1} \mathbb{1} .
$$

The mean duration of an idle period is given, using (3.16) and (3.19) by

$$
\mathbb{E}(I P)=\left(\pi^{-}, 0\right)\left(-A^{\prime}\right)^{-1} \mathbb{1}=\pi^{-}\left(-Q_{--}\right)^{-1} R_{-}^{-1}\left(I+T_{-0}\left(-T_{00}\right)^{-1}\right) \mathbb{1} .
$$

The mean durations $\mathbb{E}(B P)$ and $\mathbb{E}(I P)$ are related by the relation

$$
\mathbb{P}\{X=0\}=\frac{\mathbb{E}(I P)}{\mathbb{E}(I P)+\mathbb{E}(B P)},
$$

where $X$ is the fluid level in stationary regime. We then have from (3.35)

$$
\pi_{\text {loss }}=\frac{\mathbb{P}\{X=0\} \mathbb{E}(V(x))}{\mathbb{E}(I P) \mathbb{E}(\chi)} .
$$

The distribution of $X$ and more precisely the quantity $\mathbb{P}\{X=0\}$ has been obtained in [10] using matrix analytic methods.

To conclude this section, we note that the matrices $\Psi(x), M(x), H(x)$ and $\Gamma(x)$ are equivalent to the matrices $G(0, x), H(0, x), H(x, x)$ in [4] and the matrix $\Psi_{0}$ in [8] respectively, and can efficiently be evaluated using the explicit expressions in [7, Corollary 2] and [8, Theorem 3] (substitute $s=0$ ) and the quadratic algorithms in $[5,6]$, and without the use of numerical inversion of the Laplace-Stieltjes transforms.

Matrices $\Psi(x), H(x)$ and $\Gamma(x)$ are expressed here in terms of matrix differential Riccati equations that can be solved using a classical method which consists in computing the exponential of matrix $C Q x$ and then in solving a linear system which parameters are blocks of matrix $\exp (C Q x)$. Matrix $M(x)$, see equation (3.11), is the solution to a system of ODE with non-constant coefficient matrix that can easily be solved numerically using a classical Runge-Kutta fourth order method available in Matlab, for instance.

\section{Conclusion}

In this chapter, we have first deeply analyzed congestion when the buffer content is described by means of a Markov modulated fluid flow model in the stationary regime. In order to obtain such performance metrics we have analyzed hitting probabilities together with the maximum peak observed within a busy period in both the infinite and finite buffer case. 
The distributions of congestion metrics, defined as the total amount of lost information or the duration of congestion within a busy period, have then been obtained. We established that these distributions are of phase-type. Finally we proposed an exact solution to compute the metric $\pi_{\text {loss }}$ as defined by Equation (1.1).

\section{References}

[1] Ahn, S. and Ramaswami, V. (2003). Fluid flow models and queues - a connection by stochastic coupling. Stochastic Models, 19(3), 325-348.

[2] Ahn, S. and Ramaswami, V. (2004). Transient analysis of fluid flow models via stochastic coupling to a queue, Stochastic Models, 20(1), 71-101.

[3] Asmussen, S. (1995). Stationary distributions for fluid flow models with or without Brownian noise. Stochastic Models, 11(1), 21-49.

[4] Bean, N. G., O'Reilly, M. M. and Taylor, P. G. (2005). Hitting probabilities and hitting times for stochastic fluid flows. Stochastic Processes and their Applications, 115, 1530-1556.

[5] Bean, N. G., O'Reilly, M. M. and Taylor, P. G. (2005). Algorithms for the first return probabilities for stochastic fluid flows. Stochastic Models, 21(1), 149-184.

[6] Bean, N. G., O'Reilly, M. M. and Taylor, P. G. (2008). Algorithms for the Laplace-Stieltjes transforms of the first return probabilities for stochastic fluid flows. Methodology and Computing in Applied Probability, 10(3), 381-408.

[7] Bean, N. G. and O'Reilly, M. M. (2008). Performance measures of a multilayer Markovian fluid model. Annals of Operations Research, 160(1), 99120.

[8] Bean, N. G., O'Reilly, M. M. and Taylor, P. G. (2009). Hitting probabilities and hitting times for stochastic fluid flows: The bounded model. Probability in the Engineering and Informational Sciences, 23, 121-147.

[9] da Silva Soares, A. and Latouche, G. (2002). Further results on the similarity between fluid queues and QBDs. In G. Latouche and P. Taylor, editors, Proc. of the 4th Int. Conf. on Matrix-Analytic Methods (MAM'4), Adelaide, Australia, 89-106, World Scientific.

[10] da Silva Soares, A. and Latouche, G. (2006). Matrix-analytic methods for fluid queues with finite buffers. Performance Evaluation, 63(4), 295-314.

[11] Guillemin, F. and Sericola, B. (2007). Stationary analysis of a fluid queue driven by some countable state space Markov chain. Methodology and Computing in Applied Probability, 9, 521-540. 
[12] Guillemin, F. and Sericola, B. (2012). On the Fluid Queue Driven by an Ergodic Birth and Death Process. Telecommunications Networks - Current Status and Future Trends, Dr. Jess Ortiz (Ed.),

[13] Neuts, M.F. (1979). A versatile Markovian point process. Journal of Applied Probability, (16)3, 764-779.

[14] O'Reilly M. M. and Palmowski, Z. (2013). Loss rates for stochastic fluid models. Performance Evaluation, 70, 593 - 606.

[15] Ramaswami, V. (1999). Matrix analytic methods for stochastic fluid flows. In D. Smith and P. Hey, editors, Proceedings of the 16th International Teletraffic Congress : Teletraffic Engineering in a Competitive World (ITC'16), Edinburgh, UK, 1019-1030, Elsevier.

[16] Ramaswami, V. (2006). Passage Times in Fluid Models with Application to Risk Processes. Methodology and Computing in Applied Probability, 8, 497-515.

[17] Remiche, M.-A. (2005). Compliance of the token-bucket model with Markovian traffic. Stochastic Models, 21, 615-630.

[18] Rogers, L. C. G. (1994). Fluid models in queueing theory and Wiener-Hopf factorization of Markov chains. Advances in Applied Probability, 4(2).

[19] Rubino, G. and Sericola, B. (1989). Accumulated reward over the $\mathrm{n}$ first operational periods in fault-tolerant computing systems. Tech. Rep. 1028, INRIA.

[20] Samuelson, A. and O'Reilly, M. and Bean, N. (2017). On the generalized reward generator for stochastic fluid models: A new equation for $\Psi$. Stochastic Models, 33(4), 495-523.

[21] Sericola, B. (2001). A finite buffer fluid queue driven by a Markovian queue. Queueing Systems - Theory and Application, 38(2), 213-220.

[22] Sericola, B. (2013). Markov Chains: Theory, Algorithms and Applications. Iste Series. Wiley. 